\title{
CHERT GEOCHEMISTRY DISCRIMINANT ANALYSIS AND K-MEANS CLUSTER ANALYSIS: RAMPART PROJECT AREA, TANANA B-1 QUADRANGLE, EAST-CENTRAL ALASKA
}

by

R.R. Reifenstuhl, R.J. Newberry, S.A. Haug, K.H. Clautice, S.A. Liss, and F.R.Weber

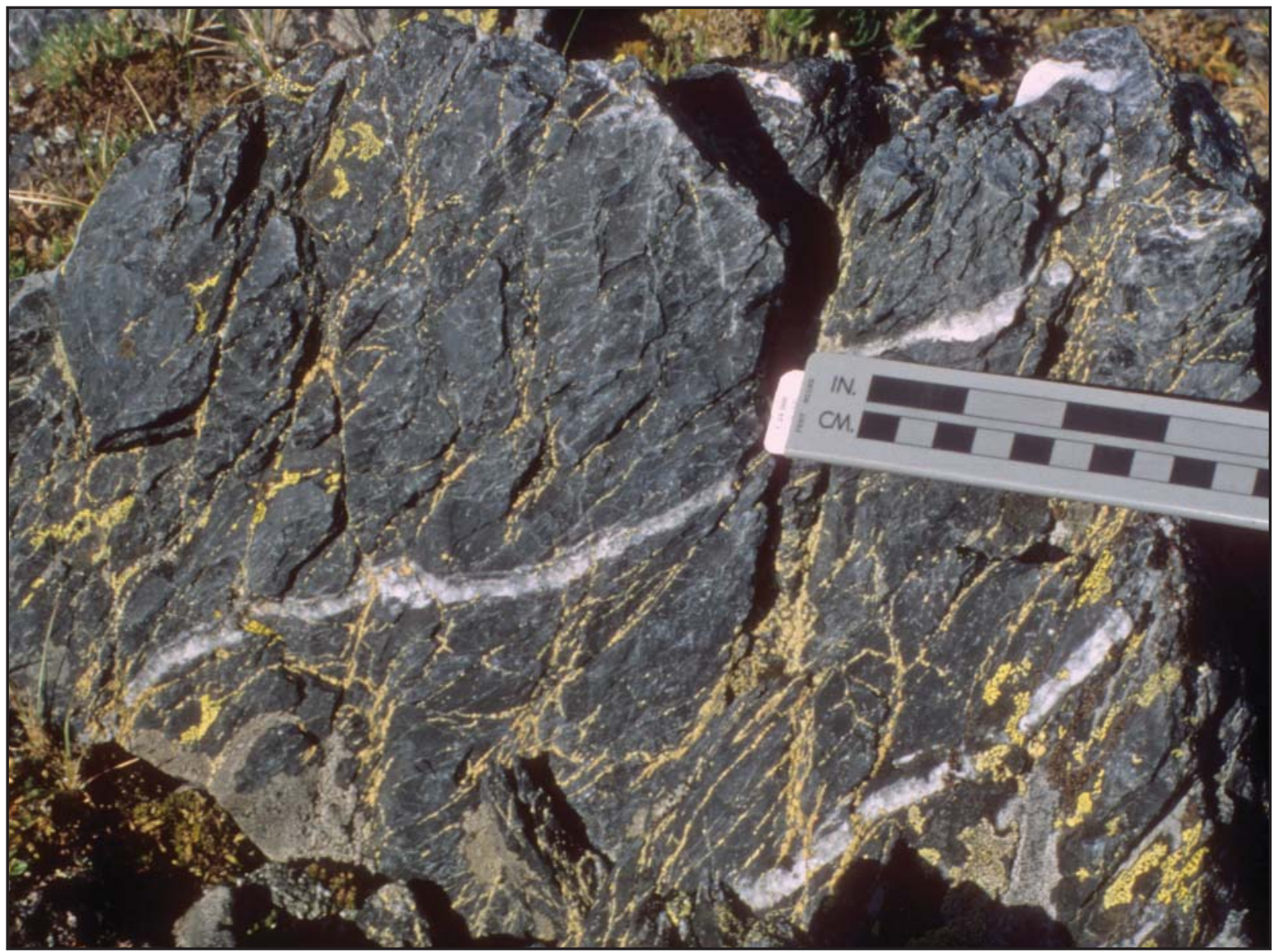

Rampart Group chert from the northeastern B-1 Quadrangle. Rock has well-developed shear fractures, some filled with yellow lichen, and cross-cutting quartz veins. These chert-rich outcrops lie close to the Victoria Creek Fault, a splay of the Tintina Fault, and are highly tectonized. Photo, Rocky Reifenstuhl

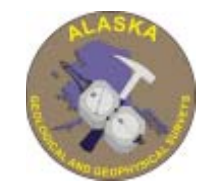

Published by

STATE OF ALASKA

DEPARTMENT OF NATURAL RESOURCES

DIVISION OF GEOLOGICAL \& GEOPHYSICAL SURVEYS 



\title{
Report of Investigations 2009-2
}

\section{CHERT GEOCHEMISTRY DISCRIMINANT ANALYSIS AND K-MEANS CLUSTER ANALYSIS: RAMPART PROJECT AREA, TANANA B-1 QUADRANGLE, EAST-CENTRAL ALASKA}

\author{
by \\ R.R. Reifenstuhl, R.J. Newberry, S.A. Haug, \\ K.H. Clautice, S.A. Liss, and F.R.Weber
}

2009

This DGGS Report of Investigations is a final report of scientific research.

It has received technical review and may be cited as an agency publication. 


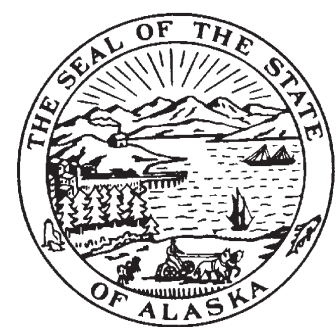

STATE OF ALASKA

Sarah Palin, Governor

DEPARTMENT OF NATURAL RESOURCES

Tom Irwin, Commissioner

DIVISION OF GEOLOGICAL \& GEOPHYSICAL SURVEYS

Robert F. Swenson, State Geologist and Director

Publications produced by the Division of Geological \& Geophysical Surveys can be examined at the following locations. To order publications, contact the Fairbanks office.
Alaska Division of Geological \&
Alaska Resource Library \& Information
Services (ARLIS) \& Geophysical Surveys
3354 College Road
Fairbanks, Alaska 99709-3707
3150 C Street, Suite 100
Anchorage, Alaska 99503
Elmer E. Rasmuson Library
University of Alaska Fairbanks
University of Alaska Anchorage Library
Fairbanks, Alaska 99775-1005
3211 Providence Drive
Anchorage, Alaska 99508

\begin{abstract}
Alaska State Library
State Office Building, 8th Floor

333 Willoughby Avenue

Juneau, Alaska 99811-0571
\end{abstract}

This publication released by the Division of Geological \& Geophysical Surveys was produced and printed in Fairbanks, Alaska, at a cost of $\$ 2$ per copy. Publication is authorized by Alaska Statute 41, which charges the division "to determine the potential of Alaskan land for production of metals, minerals, fuels, and geothermal resources; the location and supplies of groundwater and construction materials; the potential geologic hazards to buildings, roads, bridges, and other installations and structures; and shall conduct such other surveys and investigations as will advance knowledge of the geology of Alaska." 


\section{CONTENTS}

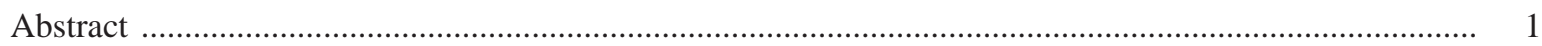

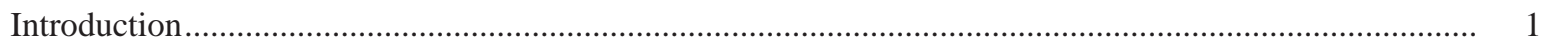

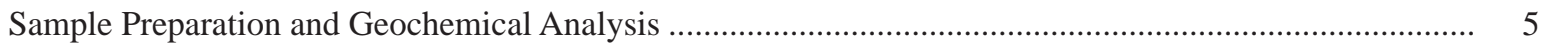

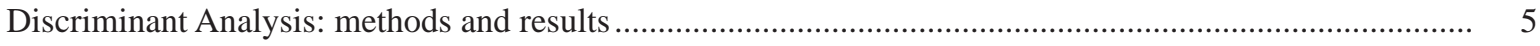

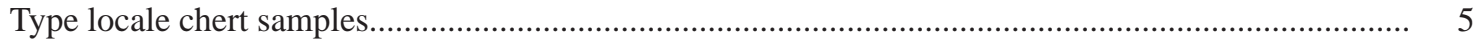

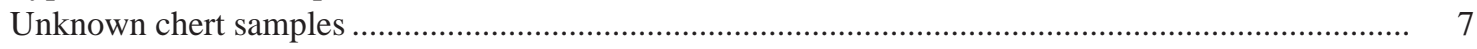

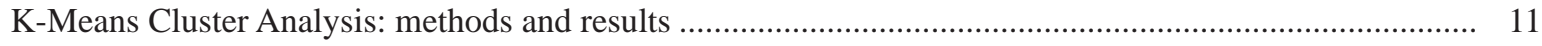

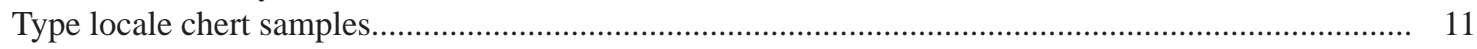

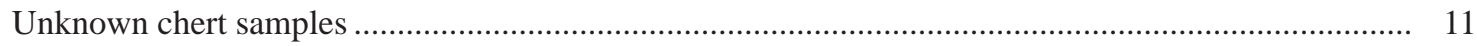

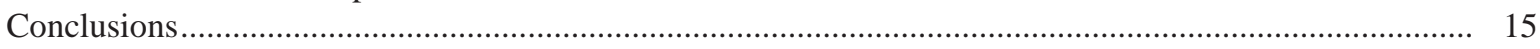

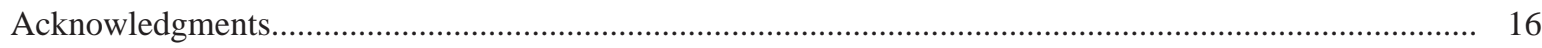

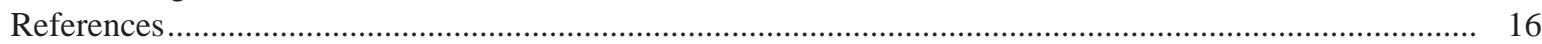

\section{FIGURES}

Figure 1. Location map for chert samples in, or adjacent to, the Tanana B-1 Quadrangle ....................... 2

2. Graph showing type locale chert samples.......................................................................

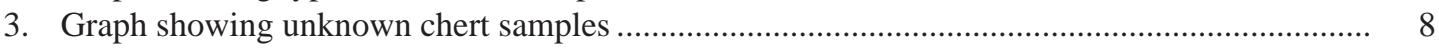

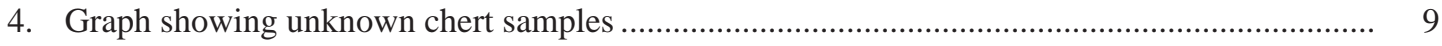

5. Plot of magnesium oxide $(\mathrm{MgO})$ versus vanadium $(\mathrm{V})$ for chert samples .............................. 15

\section{TABLES}

Table 1. Geochemical, location and sample data for cherts from the Rampart project area .................... 3

2. Discriminant analysis applied to the type locale cherts produces a 100 percent correlation between the type locale units and the predicted group based on the statistical analysis ............. 5

3. Type locale data unit predictions based on discriminant analysis ........................................... 7

4. The hypothesized rock unit for each chert sample shown next to the predicted unit generated by the discriminant analysis.

5. K-means cluster analysis of type locale chert data, final Cluster Centers ............................... 12

6. K-means cluster analysis of original data, chert samples from unknown units, final Cluster Centers

7. K-means cluster analysis of discriminant analysis data, final Cluster Centers......................... 14 



\title{
CHERT GEOCHEMISTRY DISCRIMINANT ANALYSIS AND K-MEANS CLUSTER ANALYSIS: RAMPART PROJECT AREA, TANANA B-1 QUADRANGLE, EAST-CENTRAL ALASKA
}

\author{
by
}

\author{
R.R. Reifenstuhl ${ }^{1}$, R.J. Newberry², S.A. Haug ${ }^{3}$, K.H. Clautice ${ }^{4}$, S.A. Liss ${ }^{5}$, and F.R.Weber ${ }^{6}$
}

\begin{abstract}
A pilot chemostratigraphy study using discriminant analysis on major oxide and minor element data from 67 chert samples in the Rampart area, southeastern Tanana and southwest Livengood quadrangles, western Yukon-Tanana Upland, Alaska, generally indicates a unique geochemical signature for the cherts of a given rock unit. Chert samples from five known type locales were used as standards of comparison:

-Livengood Dome Chert (Ordovician),

-Amy Creek unit (Proterozoic to early Paleozoic),

-Rampart Group (Mississippian to Triassic),

-Troublesome Creek unit (Devonian), and

-Permian-Triassic clastic unit (associated with the Triassic-dated gabbro).

Samples from the above units were compared to chert from Tanana B-1 area units of unknown or uncertain affinity. We have determined that discriminant analysis of chert geochemistry can assign chert profiles to specific units with only minor exceptions, and is useful in geologic mapping of the Tanana B-1 Quadrangle (Reifenstuhl and others, 1997).
\end{abstract}

\section{INTRODUCTION}

Our chemostratigraphic pilot study, completed in 1997, used statistical analyses techniques (discussed below) that were the standard in the 1990s. In 2008, similar statistical manipulations are currently more widely recognized and considered the standard. These include principal component analysis (PCA), in which sample groups are based on minimizing the chemical variance among the data and then testing for a statistical difference between the groups. Since we had five known rock units, our data, and statistical manipulation of that data, remains a valid treatment particularly for this pilot study and remains unchanged from our original 1997 study.

This pilot study addresses the problem of correlating rock units that lack fossils, lack stratigraphic continuity, or are highly structurally disrupted, but that are chertbearing. In the Tanana B-1 Quadrangle (fig. 1), our mapping revealed that in some cases the same chert-bearing units previously had been mapped as Baldry terrain (Dover, 1994) and Livengood Dome Chert (Chapman and others, 1982).
We set out to test whether the cherts from our five chert-bearing rock units in the Tanana B-1 Quadrangle have some unique and definable geochemical signature, and whether the chert signature could be used to correlate units across large distances in a structurally complex or structurally dismembered area. This chert geochemistry technique is not an established method and our pilot study was intended to test its viability.

Discriminant and K-means cluster analyses were applied to 67 chert samples as a pilot study to determine whether statistical analysis can aid in differentiating chert samples from different lithologic units. The chert samples were collected from the Rampart area, southeastern Tanana Quadrangle, and southwestern Livengood Quadrangle, western Yukon-Tanana Upland, Alaska. This application of statistical analysis was useful in the geologic mapping of the Tanana B-1 Quadrangle (Reifenstuhl and others, 1997). Other geological, geochemical, and geochronological information for the Tanana B-1 Quadrangle are available as part of the

${ }^{1}$ Alaska Division of Geological \& Geophysical Surveys, 3354 College Rd., Fairbanks, Alaska 99709-3707

${ }^{2}$ University of Alaska, Department of Geology \& Geophysics, P.O. Box 755780, Fairbanks, Alaska 99775-5780

${ }^{3} 1908$ Florida Avenue NW, Apt. 205, Washington, DC 20009-1213

${ }^{4}$ P.O. Box 83628, Fairbanks, Alaska 99708-3628

${ }^{5} 2749$ Goldstream Road, Fairbanks, Alaska 99709-6066

${ }^{6}$ P.O. Box 80745, Fairbanks, Alaska 99708-0745 


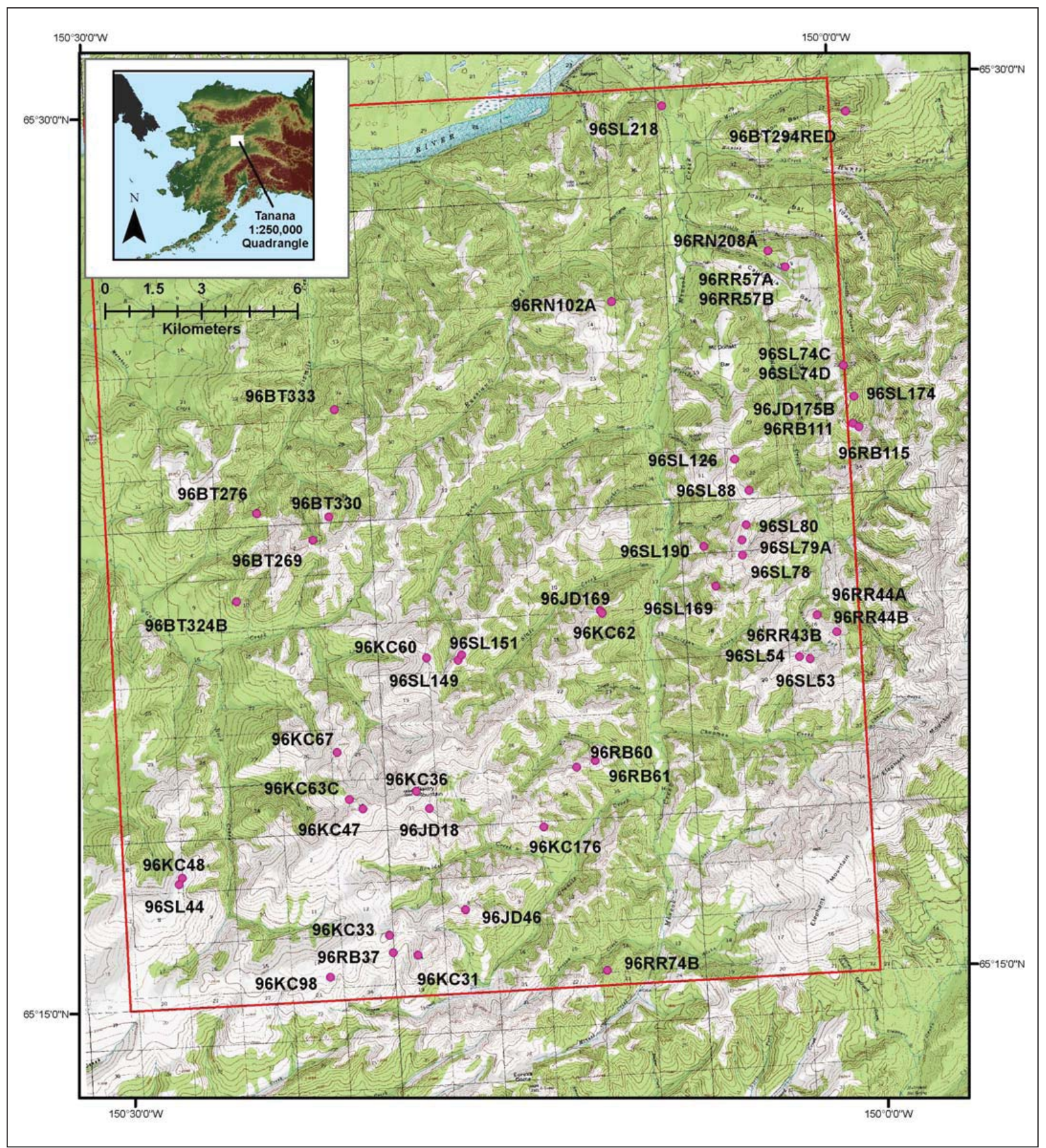

Figure 1. Location map for chert samples in, or adjacent to, the Tanana B-1 Quadrangle (red outline). See text and table 1 for details. Base from U.S. Geological Survey quadrangle maps. 
Table 1. Geochemical, location and sample data for chert samples from the Rampart project area. See figure 1 for map location of each chert sample that is in, or adjacent to, the Tanana B-1 Quadrangle.

\begin{tabular}{|c|c|c|c|c|c|c|c|c|c|c|c|c|c|c|c|c|c|c|c|c|c|c|}
\hline Sample & Rock unit/Comments & $\begin{array}{c}\text { Latitude (dec. } \\
\text { degree) }\end{array}$ & $\begin{array}{c}\text { Longitude (dec. } \\
\text { degree) }\end{array}$ & $\begin{array}{c}\text { ppm } \\
\text { Ba }\end{array}$ & $\mathrm{Ce}$ & $\mathbf{R b}$ & $\mathrm{Sr}$ & $\mathbf{Y}$ & $\mathrm{Zr}$ & $\mathrm{Cr}$ & $\mathbf{V}$ & La & $\begin{array}{l}\mathrm{wt} \% \\
\mathrm{SiO}_{2} \\
\end{array}$ & $\mathrm{TiO}_{2}$ & $\mathrm{Al}_{2} \mathrm{O}_{3}$ & $\mathrm{Fe}_{2} \mathrm{O}_{3}{ }^{*}$ & MnO & MgO & $\mathrm{CaO}$ & $\mathrm{Na}_{2} \mathrm{O}$ & $\mathrm{K}_{2} \mathrm{O}$ & $\mathbf{P}_{2} \mathbf{O}_{5}$ \\
\hline 96BT294RED & Rampart Group & 65.49028 & -149.9889 & 5 & 31 & 0 & 29 & 10 & 40 & 12 & 24 & 7 & 89.4 & 0.19 & 4.78 & 1.80 & 0.04 & 1.06 & 0.31 & 2.33 & 0.03 & 0.03 \\
\hline 96ВТ324В & Rampart Group & 65.36295175 & -150.4151728 & 259 & 18 & 8 & 7 & 4 & 23 & 0 & 19 & 1 & 90.5 & 0.13 & 3.58 & 1.94 & 0.03 & 2.07 & 0.00 & 0.47 & 0.31 & 0.01 \\
\hline 96ВТЗ333 & Rampart Group & 65.41507949 & -150.3430184 & 30 & 0 & 1 & 43 & 6 & 36 & 7 & 37 & 3 & 88.3 & 0.17 & 4.79 & 0.75 & 0.04 & 0.72 & 2.69 & 2.50 & 0.03 & 0.02 \\
\hline 96SL218 & Rampart Group & 65.49487674 & -150.1120449 & 124 & 0 & 9 & 8 & 4 & 11 & 1 & 15 & 0 & 96.1 & 0.08 & 1.67 & 0.52 & 0.04 & 0.61 & 0.06 & 0.03 & 0.31 & 0.02 \\
\hline 96BT269 & Rampart Group & 65.37894448 & -150.3619934 & 7,166 & 9 & 18 & 69 & 48 & 84 & 173 & 180 & 16 & 81.7 & 0.30 & 6.50 & 2.52 & 0.09 & 3.70 & 0.66 & 0.93 & 1.09 & 0.54 \\
\hline 96BT276 & Rampart Group & 65.38716239 & -150.3984426 & 363 & 9 & 26 & 40 & 13 & 35 & 0 & 72 & 7 & 85.3 & 0.27 & 5.81 & 3.83 & 0.39 & 1.84 & 0.10 & 0.85 & 1.17 & 0.04 \\
\hline 96ВТЗ30 & Rampart Group & 65.38520654 & -150.3504882 & 2,881 & 2 & 42 & 22 & 20 & 70 & 15 & 60 & 8 & 81.0 & 0.37 & 8.08 & 4.43 & 0.40 & 2.44 & 0.72 & 0.21 & 1.30 & 0.06 \\
\hline 96RN102A & Rampart Group & 65.44099 & -150.1531 & 2,607 & 17 & 70 & 22 & 10 & 112 & 8 & 116 & 5 & 76.4 & 0.45 & 10.89 & 4.35 & 0.12 & 3.75 & 0.06 & 0.72 & 2.25 & 0.05 \\
\hline 96RN208A & Rampart Group & 65.4525957 & -150.04654 & 1,254 & 4 & 17 & 79 & 14 & 100 & 14 & 168 & 8 & 79.2 & 0.42 & 9.12 & 3.89 & 0.05 & 3.77 & 0.94 & 2.76 & 0.49 & 0.06 \\
\hline 96RR57A & Rampart Group & 65.44784753 & -150.0355774 & 1,311 & 4 & 25 & 5 & 10 & 59 & 5 & 30 & 3 & 89.1 & 0.17 & 5.48 & 1.69 & 0.10 & 2.32 & 0.02 & 0.03 & 0.74 & 0.02 \\
\hline 96RR57B & Rampart Group & 65.44784753 & -150.0355774 & 2,081 & 36 & 82 & 10 & 14 & 88 & 6 & 125 & 10 & 80.1 & 0.36 & 10.06 & 3.97 & 0.11 & 3.79 & 0.29 & 0.05 & 2.00 & 0.05 \\
\hline 96RN12A-C1 & Amy Creek Dolomite/Livengood Quadrangle & 65.5 & -148.725 & 1,163 & 4 & 10 & 23 & 4 & 12 & 0 & 74 & 0 & 96.2 & 0.04 & 1.50 & 0.85 & 0.01 & 0.26 & 0.00 & 0.09 & 0.30 & 0.03 \\
\hline 96RN12A-C2 & Amy Creek Dolomite/Livengood Quadrangle & 65.5 & -148.725 & 1,193 & 8 & 10 & 25 & 5 & 13 & 0 & 81 & 1 & 95.7 & 0.05 & 1.60 & 0.88 & 0.01 & 0.28 & 0.00 & 0.09 & 0.33 & 0.03 \\
\hline 96RN14A & Amy Creek Dolomite/Livengood Quadrangle & 65.49167 & -148.6167 & 425 & 1 & 8 & 6 & 4 & 10 & 0 & 174 & 0 & 97.8 & 0.03 & 1.07 & 0.40 & 0.00 & 0.14 & 0.00 & 0.00 & 0.19 & 0.23 \\
\hline 96RN14B & Amy Creek Dolomite/Livengood Quadrangle & 65.49167 & -148.6167 & 1,194 & 2 & 5 & 16 & 2 & 10 & 0 & 31 & 0 & 97.7 & 0.03 & 0.67 & 0.33 & 0.00 & 0.03 & 0.00 & 0.00 & 0.15 & 0.06 \\
\hline 96RN14C & Amy Creek Dolomite/Livengood Quadrangle & 65.49167 & -148.6167 & 4,938 & 15 & 10 & 134 & 48 & 25 & 0 & 157 & 15 & 96.4 & 0.11 & 2.28 & 0.24 & 0.00 & 0.19 & 0.00 & 0.00 & 0.22 & 0.76 \\
\hline 96КС317 & $\begin{array}{l}\text { Livengood Dome Chert equivalent or Road River Formation } \\
\text { (Yukon Territory) }\end{array}$ & 64.467 & -138.333 & 205 & 12 & 4 & 12 & 2 & 5 & 0 & 12 & 1 & 96.8 & 0.03 & 1.01 & 0.86 & 0.10 & 0.45 & 0.01 & 0.05 & 0.12 & 0.01 \\
\hline 96RN13B & Livengood Dome Chert rock pit / Livengood Quadrangle & 65.5333 & -148.8417 & 74 & 2 & 5 & 17 & 1 & 6 & 1 & 7 & 0 & 97.8 & 0.01 & 0.45 & 0.55 & 0.09 & 0.05 & 0.00 & 0.09 & 0.12 & 0.01 \\
\hline 96RN13C & Livengood Dome Chert rock pit / Livengood Quadrangle & 65.5333 & -148.8417 & 119 & 10 & 4 & 22 & 1 & 6 & 2 & 9 & 1 & 97.2 & 0.01 & 0.82 & 0.71 & 0.32 & 0.30 & 0.05 & 0.08 & 0.13 & 0.01 \\
\hline 96RN13D2 & Livengood Dome Chert rock pit / Livengood Quadrangle & 65.5333 & -148.8417 & 123 & 25 & 27 & 40 & 11 & 47 & 11 & 6 & 7 & 96.1 & 0.27 & 1.59 & 1.89 & 0.06 & 0.39 & 0.09 & 0.03 & 0.69 & 0.11 \\
\hline 96RN13E & Livengood Dome Chert rock pit / Livengood Quadrangle & 65.5333 & -148.8417 & 118 & 9 & 4 & 22 & 3 & 9 & 1 & 8 & 1 & 97.4 & 0.01 & 0.77 & 0.29 & 0.01 & 0.09 & 0.00 & 0.09 & 0.13 & 0.02 \\
\hline 96RN15 & Livengood Dome Chert rock pit / Livengood Quadrangle & 65.09 & -150.29 & 23 & 6 & 0 & 1 & 0 & 0 & 0 & 4 & 1 & 99.1 & 0.00 & 0.00 & 0.03 & 0.00 & 0.04 & 0.02 & 0.01 & 0.00 & 0.00 \\
\hline 88AWR52 & $\begin{array}{l}\text { Devonian age-Troublesome Creek Unit, Livengood } \\
\text { Quadrangle }\end{array}$ & $\ldots$ & $\ldots$ & 89 & 16 & 5 & 104 & 3 & 11 & 9 & 20 & 3 & 93.4 & 0.03 & 0.2 & 0.99 & 0.06 & 1.39 & 2.07 & 0.72 & 0.02 & 0.01 \\
\hline 88AWR65D & $\begin{array}{l}\text { Devonian age-Troublesome Creek Unit, Livengood } \\
\text { Quadrangle }\end{array}$ & $-\cdot$ & 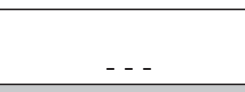 & 2,164 & 1 & 6 & 15 & 6 & 21 & 21 & 39 & 5 & 94.6 & 0.08 & 0.3 & 1.74 & 0.02 & 1.20 & 0.13 & 0.08 & 0.20 & 0.02 \\
\hline 96RR43B & Triassic age chert & 65.34506431 & -150.0157816 & 186 & 68 & 36 & 35 & 21 & 220 & 35 & 104 & 21 & 75.6 & 0.66 & 10.17 & 8.49 & 0.22 & 2.63 & 0.20 & 0.81 & 1.02 & 0.13 \\
\hline 96RR44A & Triassic age chert & 65.35008196 & -150.0280757 & 202 & 0 & 16 & 9 & 10 & 50 & 15 & 64 & 5 & 90.6 & 0.20 & 3.64 & 2.96 & 0.02 & 1.31 & 0.00 & 0.18 & 0.41 & 0.05 \\
\hline 96RR44B & Triassic age chert & 65.35008196 & -150.0280757 & 490 & 22 & 22 & 14 & 16 & 70 & 13 & 53 & 9 & 86.0 & 0.24 & 5.49 & 5.43 & 0.21 & 1.89 & 0.03 & 0.29 & 0.62 & 0.03 \\
\hline 96SL53 & Triassic age chert & 65.33801531 & -150.0346592 & 393 & 11 & 27 & 17 & 11 & 60 & 42 & 75 & 21 & 87.1 & 0.26 & 5.81 & 3.30 & 0.05 & 1.66 & 0.01 & 0.66 & 0.75 & 0.03 \\
\hline 96SL54 & Triassic age chert & 65.33878423 & -150.0415366 & 285 & 30 & 1 & 8 & 4 & 111 & 4 & 35 & 4 & 93.6 & 0.12 & 1.73 & 2.49 & 0.06 & 0.89 & 0.07 & 0.06 & 0.01 & 0.04 \\
\hline 96JD169 & Livengood Dome Chert? & 65.35475869 & -150.1724594 & 109 & 0 & 4 & 6 & 3 & 4 & 0 & 8 & 0 & 98.5 & 0.03 & 0.94 & 0.30 & 0.05 & 0.20 & 0.00 & 0.02 & 0.14 & 0.03 \\
\hline 96JD46 & Livengood Dome Chert? & 65.27337761 & -150.2739151 & 17 & 11 & 2 & 2 & 0 & 2 & 0 & 5 & 1 & 98.4 & 0.01 & 0.24 & 0.16 & 0.00 & 0.07 & 0.00 & 0.00 & 0.07 & 0.01 \\
\hline 96КС 33 & Livengood Dome Chert? & 65.2674011 & -150.3253691 & 71 & 16 & 6 & 2 & 1 & 3 & 1 & 5 & 1 & 98.2 & 0.02 & 0.69 & 0.14 & 0.00 & 0.13 & 0.00 & 0.01 & 0.18 & 0.01 \\
\hline $96 \mathrm{KC} 48$ & Livengood Dome Chert? & 65.28652011 & -150.461303 & 67 & 0 & 5 & 2 & 2 & 4 & 0 & 11 & 0 & 98.5 & 0.02 & 1.12 & 0.21 & 0.01 & 0.18 & 0.00 & 0.01 & 0.20 & 0.02 \\
\hline $96 \mathrm{KC} 60$ & Livengood Dome Chert? & 65.3443815 & -150.2904524 & 51 & 17 & 18 & 4 & 1 & 14 & 5 & 19 & 1 & 94.5 & 0.08 & 2.35 & 1.45 & 0.05 & 0.38 & 0.00 & 0.03 & 0.61 & 0.01 \\
\hline 96КС 62 & Livengood Dome Chert? & 65.35414773 & -150.1716684 & 62 & 32 & 4 & 4 & 2 & 3 & 1 & 7 & 1 & 97.7 & 0.02 & 0.79 & 0.19 & 0.01 & 0.11 & 0.00 & 0.00 & 0.17 & 0.02 \\
\hline 96КС67 & Livengood Dome Chert? & 65.31932678 & -150.3534493 & 52 & 11 & 8 & 5 & 2 & 9 & 4 & 14 & 1 & 95.9 & 0.06 & 2.16 & 0.24 & 0.00 & 0.27 & 0.00 & 0.03 & 0.40 & 0.01 \\
\hline 96RB37 & Livengood Dome Chert? & 65.26250054 & -150.3234956 & 61 & 16 & 3 & 3 & 1 & 2 & 0 & 7 & 1 & 97.9 & 0.02 & 0.63 & 0.14 & 0.00 & 0.13 & 0.00 & 0.01 & 0.16 & 0.01 \\
\hline 96RB61 & Livengood Dome Chert? & 65.31304 & -150.182 & 37 & 1 & 1 & 3 & 1 & 2 & 1 & 5 & 0 & 99.1 & 0.01 & 0.31 & 0.09 & 0.00 & 0.06 & 0.00 & 0.00 & 0.04 & 0.02 \\
\hline
\end{tabular}

*All iron reported as Fe2O3 
Table 1 - continued. Geochemical, location and sample data for chert samples from the Rampart project area. See figure 1 for map location of each chert sample that is in, or adjacent to, the Tanana B-1 Quadrangle.

\begin{tabular}{|c|c|c|c|c|c|c|c|c|c|c|c|c|c|c|c|c|c|c|c|c|c|c|}
\hline Sample & Rock unit/Comments & $\begin{array}{c}\text { Latitude (dec. } \\
\text { degree) }\end{array}$ & $\begin{array}{c}\text { Longitude (dec. } \\
\text { degree) }\end{array}$ & $\begin{array}{c}\text { ppm } \\
\text { Ba }\end{array}$ & $\mathrm{Ce}$ & $\mathbf{R b}$ & $\mathrm{Sr}$ & $\mathbf{Y}$ & $\mathrm{Zr}$ & $\mathrm{Cr}$ & $\mathbf{v}$ & $\mathrm{La}$ & $\begin{array}{l}\mathrm{wt}^{\mathrm{t}} \% \\
\mathrm{SiO}_{2} \\
\end{array}$ & $\mathrm{TiO}_{2}$ & $\mathrm{Al}_{2} \mathrm{O}_{3}$ & $\mathrm{Fe}_{2} \mathrm{O}_{3}{ }^{*}$ & MnO & MgO & $\mathrm{CaO}$ & $\mathrm{Na}_{2} \mathrm{O}$ & $\mathrm{K}_{2} \mathrm{O}$ & $\mathbf{P}_{2} \mathrm{O}_{5}$ \\
\hline 96SL126 & Livengood Dome Chert? & 65.39488321 & -150.0769903 & 165 & 1 & 16 & 3 & 3 & 14 & 2 & 21 & 1 & 95.2 & 0.10 & 2.53 & 0.71 & 0.04 & 0.26 & 0.00 & 0.00 & 0.52 & 0.04 \\
\hline 96SL149 & Livengood Dome Chert? & 65.34319707 & -150.2694664 & 64 & 15 & 6 & 9 & 1 & 2 & 1 & 8 & 1 & 97.4 & 0.03 & 0.99 & 0.34 & 0.03 & 0.20 & 0.00 & 0.01 & 0.23 & 0.01 \\
\hline 96SL151 & Livengood Dome Chert? & 65.34466422 & -150.2667081 & 55 & 3 & 7 & 8 & 2 & 4 & 2 & 12 & 0 & 97.1 & 0.03 & 1.08 & 0.25 & 0.03 & 0.20 & 0.00 & 0.01 & 0.25 & 0.01 \\
\hline 96SL190 & Livengood Dome Chert? & 65.37095128 & -150.1010443 & 146 & 25 & 7 & 4 & 2 & 10 & 6 & 14 & 1 & 97.1 & 0.06 & 1.29 & 0.35 & 0.01 & 0.14 & 0.00 & 0.01 & 0.27 & 0.01 \\
\hline 96SL44 & Livengood Dome Chert? & 65.28474317 & -150.4636932 & 19 & 11 & 4 & 2 & 1 & 1 & 0 & 7 & 1 & 98.3 & 0.01 & 0.73 & 0.09 & 0.00 & 0.13 & 0.00 & 0.00 & 0.15 & 0.02 \\
\hline 96SL80 & Livengood Dome Chert? & 65.3763367 & -150.0718583 & 32 & 36 & 0 & 3 & 1 & 1 & 1 & 3 & 7 & 99.1 & 0.00 & 0.00 & 0.09 & 0.00 & 0.23 & 0.25 & 0.01 & 0.00 & 0.05 \\
\hline 96SL88 & Livengood Dome Chert? & 65.38592956 & -150.0687309 & 58 & 21 & 5 & 4 & 1 & 3 & 0 & 8 & 1 & 98.2 & 0.02 & 0.83 & 0.15 & 0.00 & 0.11 & 0.00 & 0.06 & 0.16 & 0.01 \\
\hline $98 \mathrm{KC} 236$ & Livengood Dome Chert? & - - & - . - & 27 & 6 & 3 & 1 & 2 & 7 & 49 & 13 & 0 & 96.5 & 0.03 & 0.87 & 1.14 & 0.25 & 0.13 & 0.03 & 0.003 & 0.06 & 0.04 \\
\hline 96JD175B & Amy Creek Dolomite? - location approximate & 65.402933 & -149.9967 & 1,013 & 1 & 11 & 20 & 9 & 17 & 0 & 112 & 6 & 96.5 & 0.10 & 2.47 & 0.76 & 0.01 & 0.39 & 0.04 & 0.01 & 0.46 & 0.23 \\
\hline 96KC176 & Amy Creek Dolomite? & 65.29523799 & -150.2185773 & 211 & 6 & 1 & 2 & 1 & 2 & 0 & 84 & 1 & 98.3 & 0.01 & 0.39 & 0.11 & 0.00 & 0.08 & 0.00 & 0.01 & 0.06 & 0.04 \\
\hline 96KC47 & Amy Creek Dolomite? & 65.30318071 & -150.3383743 & 119 & 8 & 4 & 3 & 4 & 12 & 0 & 126 & 1 & 98.2 & 0.03 & 0.72 & 0.25 & 0.00 & 0.12 & 0.00 & 0.01 & 0.15 & 0.01 \\
\hline 96КС98 & Amy Creek Dolomite? & 65.25656513 & -150.3661935 & 826 & 9 & 22 & 8 & 2 & 20 & 0 & 189 & 1 & 93.5 & 0.12 & 4.28 & 0.62 & 0.00 & 0.85 & 0.03 & 0.02 & 0.93 & 0.05 \\
\hline 96RB111 & Amy Creek Dolomite? & 65.402933 & -149.9967 & 286 & 6 & 3 & 19 & 4 & 5 & 3 & 55 & 1 & 97.6 & 0.03 & 0.71 & 0.41 & 0.00 & 0.10 & 0.26 & 0.01 & 0.12 & 0.20 \\
\hline 96RB121 & $\begin{array}{l}\text { Amy Creek Dolomite?/Tanana A-1 Quadrangle, S19 T5N } \\
\text { R14W }\end{array}$ & $\cdots$ & $\cdots$ & 101 & 7 & 25 & 8 & 5 & 21 & 1 & 93 & 1 & 93.6 & 0.11 & 4.80 & 0.90 & 0.01 & 0.62 & 0.00 & 0.01 & 0.88 & 0.08 \\
\hline 96RB 60 & Amy Creek Dolomite? & 65.31141977 & -150.1945329 & 62 & 11 & 5 & 4 & 3 & 6 & 0 & 75 & 1 & 98.2 & 0.03 & 0.73 & 0.20 & 0.00 & 0.15 & 0.00 & 0.01 & 0.18 & 0.01 \\
\hline 96SL169 & Amy Creek Dolomite? & 65.35969884 & -150.0946873 & 375 & 40 & 10 & 17 & 11 & 12 & 5 & 76 & 6 & 95.8 & 0.08 & 2.03 & 0.40 & 0.00 & 0.35 & 0.09 & 0.00 & 0.45 & 0.12 \\
\hline 96SL74C & Amy Creek Dolomite? & 65.41936722 & -150.0004797 & 38 & 2 & 4 & 5 & 2 & 10 & 0 & 57 & 1 & 97.9 & 0.03 & 0.71 & 0.24 & 0.00 & 0.13 & 0.00 & 0.01 & 0.14 & 0.02 \\
\hline 96SL78 & Amy Creek Dolomite? & 65.36792277 & -150.0756257 & 231 & 1 & 6 & 60 & 8 & 15 & 0 & 61 & 0 & 96.1 & 0.08 & 2.69 & 0.07 & 0.00 & 0.08 & 0.00 & 0.22 & 0.19 & 0.01 \\
\hline 96SL79A & Amy Creek Dolomite? & 65.37218449 & -150.0752668 & 47 & 36 & 5 & 2 & 5 & 2 & 0 & 54 & 1 & 97.8 & 0.02 & 0.72 & 0.25 & 0.00 & 0.12 & 0.00 & 0.00 & 0.20 & 0.01 \\
\hline 96JD119 & $\begin{array}{l}\text { Rock unit unknown; approximate location only: Tanana A-1 } \\
\text { Quadrangle }\end{array}$ & -.. & $\cdots$ & 645 & 7 & 13 & 7 & 3 & 17 & 5 & 22 & 3 & 96.2 & 0.10 & 2.64 & 0.43 & 0.01 & 0.38 & 0.00 & 0.01 & 0.53 & 0.02 \\
\hline 96JD18 & Not Amy Creek Dolomite & 65.30226348 & -150.294146 & 88 & 10 & 25 & 2 & 2 & 26 & 16 & 27 & 4 & 88.8 & 0.16 & 5.50 & 1.56 & 0.02 & 1.72 & 0.00 & 0.10 & 1.14 & 0.01 \\
\hline 96КС31 & Amy Creek Dolomite? apatite rich! & 65.26140029 & -150.3071416 & 87 & 1 & 2 & 84 & 45 & 6 & 0 & 33 & 19 & 92.8 & 0.01 & 0.28 & 0.13 & 0.00 & 0.13 & 4.14 & 0.02 & 0.06 & 2.92 \\
\hline $96 \mathrm{KC} 36$ & Amy Creek Dolomite? apatite rich! & 65.30724092 & -150.3022135 & 178 & 44 & 14 & 121 & 90 & 25 & 0 & 23 & 40 & 80.5 & 0.11 & 2.63 & 0.52 & 0.00 & 0.85 & 7.83 & 0.07 & 0.60 & 6.40 \\
\hline $96 \mathrm{KC} 63 \mathrm{C}$ & Not Amy Creek Dolomite or Devonian age unit & 65.30602672 & -150.3469967 & 62 & 23 & 16 & 5 & 2 & 19 & 11 & 19 & 1 & 94.5 & 0.10 & 2.85 & 0.70 & 0.01 & 0.72 & 0.00 & 0.17 & 0.53 & 0.01 \\
\hline 96RB115 & Livengood Dome Chert? Near sample 96SL174 & 65.401803 & -149.9927 & 118 & 13 & 9 & 2 & 2 & 9 & 5 & 20 & 1 & 94.2 & 0.07 & 2.39 & 1.38 & 0.05 & 1.15 & 0.00 & 0.04 & 0.40 & 0.01 \\
\hline 96RB130 & $\begin{array}{l}\text { Devonian? or Triassic? SW13/4 S19 T5N R13W, Tanana A-1 } \\
\text { Quadrangle }\end{array}$ & $\ldots$ & $\ldots$ & 474 & 14 & 14 & 14 & 11 & 18 & 1 & 38 & 3 & 94.8 & 0.11 & 2.76 & 1.14 & 0.01 & 0.70 & 0.18 & 0.01 & 0.63 & 0.19 \\
\hline 96RR74B & Not Livengood Dome Chert & 65.2542 & -150.1817 & 130 & 65 & 32 & 23 & 15 & 35 & 10 & 34 & 8 & 90.6 & 0.18 & 4.66 & 1.09 & 0.02 & 1.07 & 0.16 & 0.00 & 1.16 & 0.15 \\
\hline 96SL174 & Livengood Dome Chert? & 65.41049168 & -149.9948409 & 156 & 8 & 13 & 2 & 2 & 12 & 1 & 22 & 3 & 95.2 & 0.09 & 2.29 & 0.60 & 0.02 & 0.65 & 0.00 & 0.01 & 0.56 & 0.02 \\
\hline 96SL74D & Amy Creek Dolomite? Livengood Dome Chert? & 65.41936722 & -150.0004797 & 50 & 4 & 9 & 2 & 2 & 14 & 3 & 23 & 2 & 94.1 & 0.09 & 2.72 & 1.41 & 0.04 & 1.05 & 0.00 & 0.00 & 0.36 & 0.01 \\
\hline
\end{tabular}

*All iron reported as Fe2O3 
Public-Data File series, report PDF 97-29. Geochemical data of all samples taken for the Rampart project are available electronically in PDF 97-29g.

Cherts are most commonly formed on the continental slope (Sue Karl, personal commun., 1997); continental slope depths range from about $200 \mathrm{~m}$ to $4,000 \mathrm{~m}$, have an average slope of 4 degrees, and currently cover some 28 million $\mathrm{km}^{2}$ (Emery, 1970).

\section{SAMPLE PREPARATION AND GEOCHEMICAL ANALYSIS}

A total of 67 chert samples were collected from the Rampart project area (fig. 1). Of those samples, 42 were collected from areas in which the unit was not known with certainty and 25 were from the following type locales: Rampart Group (7), Amy Creek unit (5), Livengood Dome Chert (6), Devonian-age Troublesome Creek unit (2), and Chert and Gabbro unit (5) of Triassic-age (232.1 \pm 4.5 Ma lead-zircon age, Weber and others, 1992). Chert samples include a wide variety and range of colors including gray, black, red, green, and tan. Globally, chert is considered a group of highly siliceous, non-clastic sedimentary rocks (Jones and Murchey, 1986), the majority of which contain up to 98 percent $\mathrm{SiO}_{2}$ (Murry and others, 1992). Samples collected for our pilot study range between 76 and 99 percent $\mathrm{SiO}_{2}$.

The collected cherts were crushed and pelletized for X-Ray Fluorescence analyses at the University of Alaska Fairbanks (using a Rigaku machine operating at $55 \mathrm{kV}$ and $35 \mathrm{~mA}$ ). The pellets were analyzed (table 1) for 19 elements: ten major-oxide elements measured in weight percent (aluminum, calcium, iron, potassium, magnesium, manganese, sodium, phosphorous, silicon, and titanium) and nine trace elements measured in parts per million (barium, cerium, chromium, lanthanum, rubidium, strontium, vanadium, yttrium, and zirconium).

\section{DISCRIMINANT ANALYSIS: METHODS AND RESULTS}

Discriminant analysis of the geochemical data was applied first to the type locale chert samples to test whether our analysis could distinguish between the five type locales, and whether each type locale showed a separate and unique signature. On the basis of the elemental characteristics of the cherts, the discriminant analysis yields five different groupings that correspond with the five different type locales (table 2). A multiple linear regression equation determines the discriminant functions (or discriminant scores) for each sample considering all 19 measured elemental compositions of each chert (SPSS for Microsoft Windows, 1997 version).

\section{TYPE LOCALE CHERT SAMPLES}

When the discriminant functions of each type locale sample are graphed, each of the five units (Livengood Dome Chert unit, Amy Creek unit chert, Rampart Group unit chert, Devonian-age Troublesome Creek unit chert, and the Triassic-age unit chert) plot in distinct and separate clusters (fig. 2). The discriminant analysis also produced a 1.0 correlation between the type locale units and the predicted units generated by the statistical analysis (table 2), thus assuring discriminant analysis is a viable means for differentiating the chert samples into the five different units.

Twelve of the 17 samples that were hypothesized during field work to be Livengood Dome chert samples are assigned to the Livengood Dome chert unit. The remaining five chert samples (KC60, KC62, KC67, SL190, SL88) are assigned to the Amy Creek chert unit.

Of the chert samples field-hypothesized to be Amy Creek chert samples, nine of the 11 are assigned to the Amy Creek chert unit. The two samples not assigned to the Amy Creek chert unit are assigned to the Livengood Dome chert unit.

Table 2. Discriminant analysis applied to the type locale cherts produces a 100 percent correlation between the type locale units and the predicted group based on the statistical analysis.

\begin{tabular}{|c|c|c|c|c|c|}
\hline \multirow[b]{2}{*}{ Unit } & \multicolumn{4}{|c|}{ Unit Classification Results } & \multirow[b]{2}{*}{$\mathbf{T}^{\mathbf{a}}$} \\
\hline & \#Cases & $\mathbf{R}^{\mathbf{a}}$ & $\mathbf{A}^{\mathbf{a}}$ & $\mathbf{D}^{\mathbf{a}}$ & \\
\hline Rampart & 11 & $100 \%$ & 0 & 0 & 0 \\
\hline Amy Ck & 5 & 0 & $100 \%$ & 0 & 0 \\
\hline Livengood & 6 & 0 & 0 & 0 & 0 \\
\hline Devonian & 2 & 0 & 0 & $100 \%$ & 0 \\
\hline Triassic & 5 & 0 & 0 & 0 & $100 \%$ \\
\hline
\end{tabular}




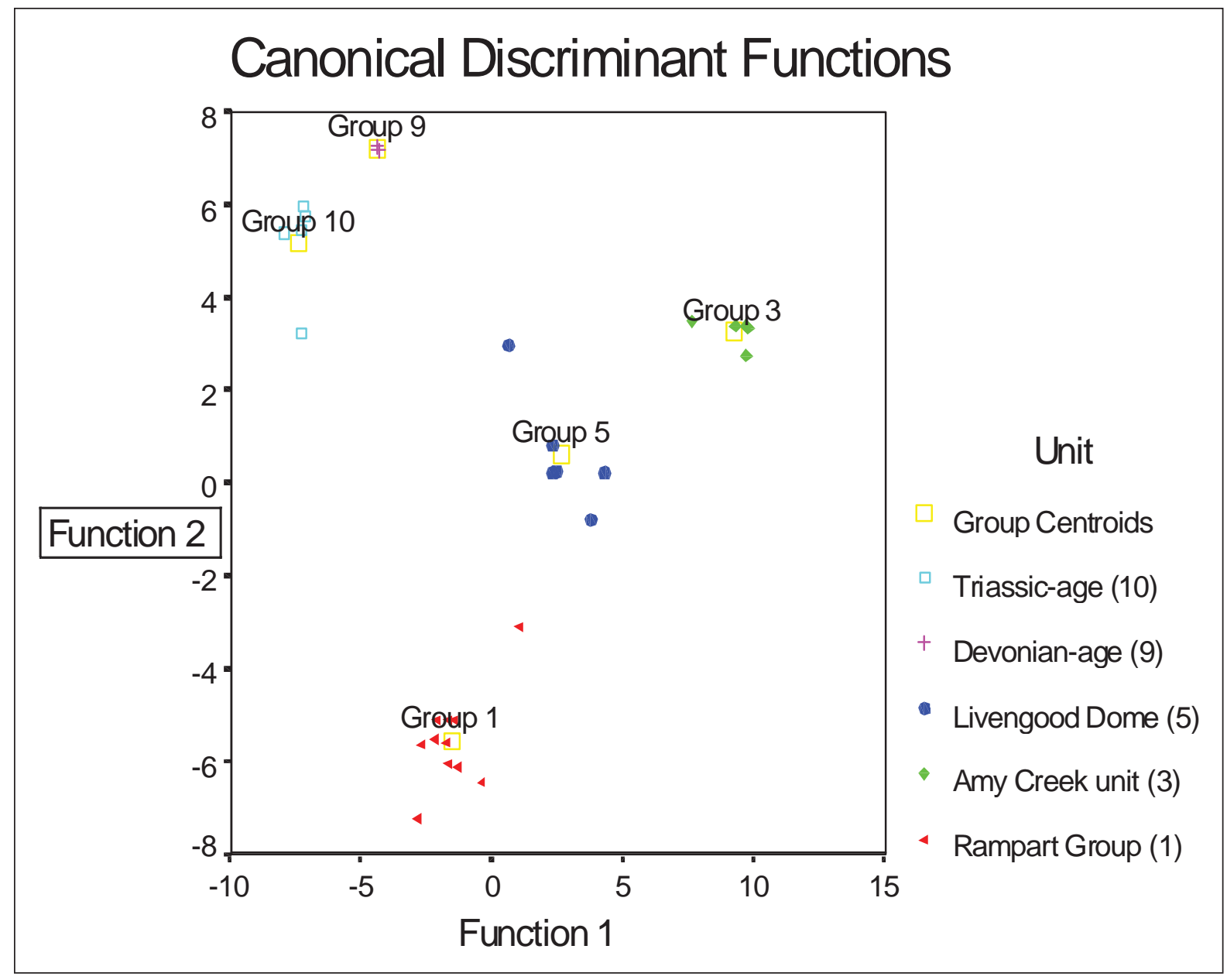

Figure 2. Type locale chert samples. See table 1 for geochemical analyses and tables 2 through 6 for statistical data.

The two chert samples statistically assigned to the Rampart Group chert unit, JD18 and RR74B, are located in the southern Tanana B-1 Quadrangle (see fig. 1) where the Rampart Group unit has not previously been mapped. The two samples most likely do not represent slivers of the Rampart Group in the south of the quadrangle. Instead, the geochemistry of these rock samples reflects the heterogeneous nature of Rampart Group cherts. This heterogeneity is seen in the K-means cluster analysis (see section K-Means cluster analysis: methods and results).

\section{UNKNOWN CHERT SAMPLES}

Discriminant analysis was then applied to the chert samples of unknown units along with the cherts from the five type locales. The program placed each unknown sample into the group it most closely resembled. The samples and statistical results are shown in table 3, where column one is the sample, column two is the hypothesized unit, as determined by the geologist in the field based on the physical characteristics and lithologic associations, column three is the predicted unit assigned by discriminant analysis, and the remaining columns the unique discriminant functions for each sample. Function graphs of the unknown cherts plotted along with the type locale cherts, based on the discriminant functions delineated group affinities (figs. 3 and 4).

The discriminant analysis of the chert samples of unknown units assigned 19 samples to Livengood Dome chert unit, 15 samples to Amy Creek chert unit, 2 samples to Rampart Group chert unit, and 2 samples to Triassic-age chert unit. (table 4).

Likewise, the two unknown chert samples (KC31 and KC36) assigned to the Triassic-age chert unit by the discriminant analysis do not represent Triassic-age cherts, but are merely most closely related to that group. KC31 and KC36 have highly anomalous $\mathrm{P}_{2} \mathrm{O}_{5}$ (2.92 weight percent and 6.4 weight percent, respectively), common to cherts formed at the base of the continental slope and thereby difficult to correlate with any of the 
Table 3. Type locale data unit predictions based on discriminant analysis

\begin{tabular}{|c|c|c|c|c|c|c|}
\hline Sample & Type Unit & Predicted & 1st Prob+ & 2nd Prob & Function1* & Function2* \\
\hline BT294red & $\mathrm{R}$ ? & $\mathrm{R}$ & $\mathrm{R} / 1$ & $\mathrm{~L} / .0000$ & -1.2758 & -6.09 \\
\hline ВТ324В & $\mathrm{R}$ ? & $\mathrm{R}$ & $\mathrm{R} / 1$ & $\mathrm{~L} / .0000$ & -2.1321 & -5.471 \\
\hline ВТЗ33 & $\mathrm{R}$ ? & $\mathrm{R}$ & $\mathrm{R} / 1$ & $\mathrm{R} / 1$ & -1.6196 & -5.9991 \\
\hline SL218 & $\mathrm{R}$ ? & $\mathrm{R}$ & $\mathrm{R} / .6262$ & L/.3738 & 1.1007 & -3.0629 \\
\hline ВТ269 & $\mathrm{R}$ & $\mathrm{R}$ & $\mathrm{R} / 1$ & $\mathrm{~L} / .0000$ & -1.6723 & -5.5661 \\
\hline BT276 & $\mathrm{R}$ & $\mathrm{R}$ & $\mathrm{R} / 1$ & $\mathrm{~L} / .0000$ & -2.6616 & -5.6 \\
\hline ВТЗ30 & $\mathrm{R}$ & $\mathrm{R}$ & $\mathrm{R} / 1$ & $\mathrm{~L} / .0000$ & -1.4291 & -5.0974 \\
\hline RN102A & $\mathrm{R}$ & $\mathrm{R}$ & $\mathrm{R} / 1$ & $\mathrm{~L} / .0000$ & -0.3812 & -6.4065 \\
\hline RN208A & $\mathrm{R}$ & $\mathrm{R}$ & $\mathrm{R} / 1$ & $\mathrm{~L} / .0000$ & -1.6358 & -5.08 \\
\hline RR57A & $\mathrm{R}$ & $\mathrm{R}$ & $\mathrm{R} / 1$ & $\mathrm{R} / 1$ & -2.7974 & -7.1917 \\
\hline RR57B & $\mathrm{R}$ & $\mathrm{R}$ & $\mathrm{R} / 1$ & $\mathrm{~L} / .0000$ & -2.0953 & -5.0872 \\
\hline RN12A-C1 & A & A & $\mathrm{A} / 1$ & $\mathrm{~L} / .0000$ & 9.7136 & 3.3984 \\
\hline RN12A-C2 & A & A & $\mathrm{A} / 1$ & $\mathrm{~L} / .0000$ & 9.7298 & 2.7673 \\
\hline RN14A & A & A & $\mathrm{A} / 1$ & $\mathrm{~L} / .0000$ & 9.3493 & 3.4139 \\
\hline RN14B & A & A & $\mathrm{A} / 1$ & $\mathrm{~L} / .0000$ & 7.6387 & 3.5277 \\
\hline RN14C & A & A & $\mathrm{A} / 1$ & $\mathrm{~A} / 1$ & 9.7896 & 3.3673 \\
\hline КС317 & $\mathrm{L}$ & $\mathrm{L}$ & $\mathrm{L} / 1$ & $\mathrm{~A} / .0000$ & 4.3324 & 0.2345 \\
\hline RN13B & $\mathrm{L}$ & $\mathrm{L}$ & $\mathrm{L} / 1$ & $\mathrm{~T} / .0000$ & 0.678 & 2.9805 \\
\hline RN13C & $\mathrm{L}$ & $\mathrm{L}$ & $\mathrm{L} / 1$ & $\mathrm{R} / .0000$ & 3.8038 & -7.685 \\
\hline RN13D2 & $\mathrm{L}$ & $\mathrm{L}$ & $\mathrm{L} / 1$ & $\mathrm{R} / .0000$ & 2.4489 & 0.2701 \\
\hline RN13E & $\mathrm{L}$ & $\mathrm{L}$ & $\mathrm{L} / 1$ & $\mathrm{R} / .0000$ & 2.3211 & 0.8341 \\
\hline RN15 & $\mathrm{L}$ & $\mathrm{L}$ & $\mathrm{L} / 1$ & $\mathrm{R} / .0000$ & 2.3391 & 0.2485 \\
\hline AWR52 & $\mathrm{D}$ & D & $\mathrm{D} / 1$ & $\mathrm{D} / 1$ & -4.3924 & 7.281 \\
\hline AWR65D & $\mathrm{D}$ & $\mathrm{D}$ & $\mathrm{D} / 1$ & $\mathrm{D} / 1$ & -4.3229 & 7.1862 \\
\hline RR43B & $\mathrm{T}$ & $\mathrm{T}$ & $\mathrm{T} / 1$ & $\mathrm{~T} / 1$ & -7.9479 & 5.4019 \\
\hline RR44A & $\mathrm{T}$ & $\mathrm{T}$ & $\mathrm{T} / 1$ & $\mathrm{~T} / 1$ & -7.2913 & 3.2383 \\
\hline RR44B & $\mathrm{T}$ & $\mathrm{T}$ & $\mathrm{T} / 1$ & $\mathrm{~T} / 1$ & -7.1312 & 5.7771 \\
\hline SL53 & $\mathrm{T}$ & $\mathrm{T}$ & $\mathrm{T} / 1$ & $\mathrm{~T} / 1$ & -7.2764 & 5.5037 \\
\hline SL54 & $\mathrm{T}$ & $\mathrm{T}$ & $\mathrm{T} / 1$ & $\mathrm{~T} / 1$ & -7.1829 & 5.99 \\
\hline
\end{tabular}

*These functions are plotted in figure 2 . 


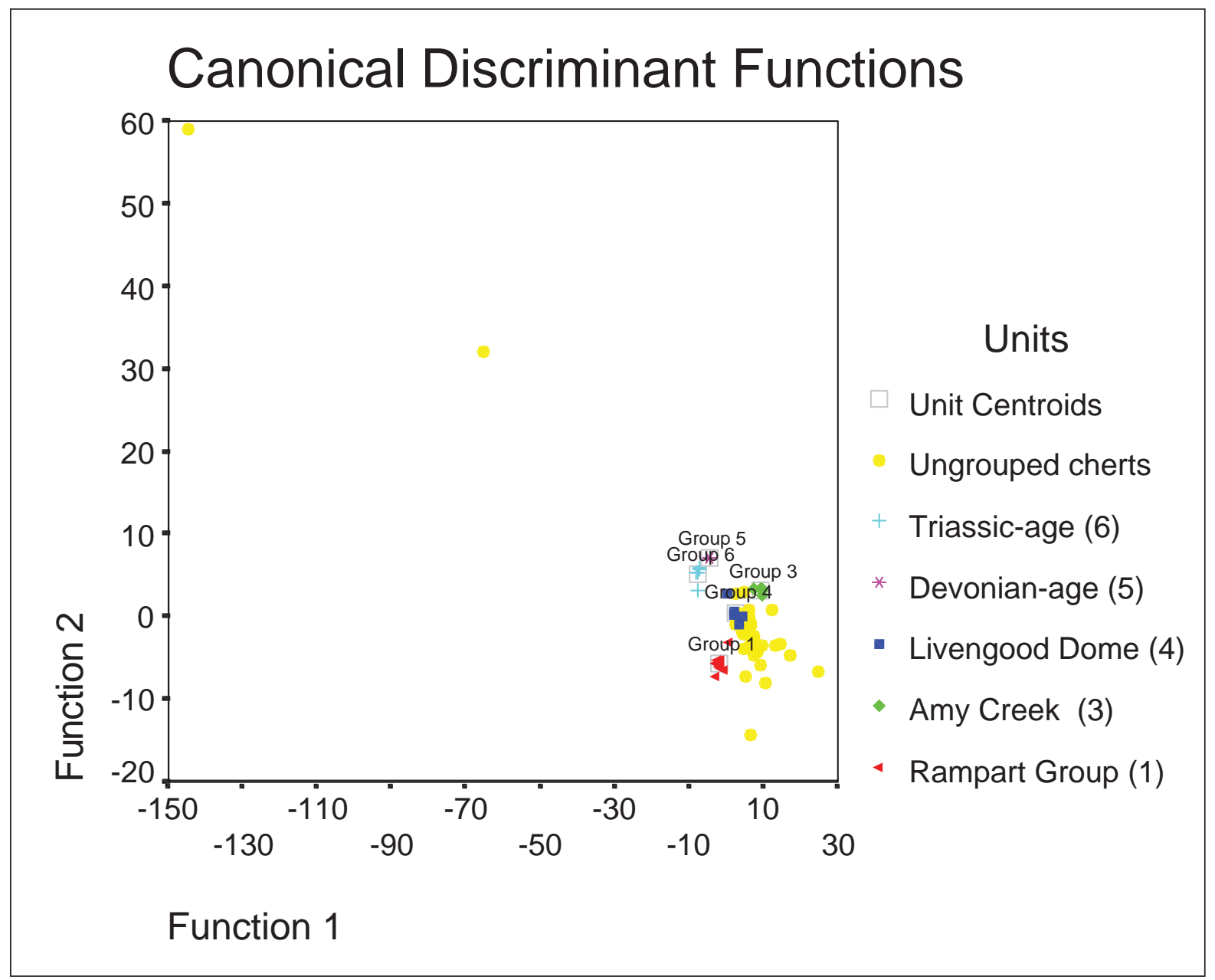

Figure 3. Unknown chert samples; each type locale unit plots separately and uniquely. Function 1 and Function 2 are generated by the discriminant analysis and are listed in table 4 for each sample. The two outliers, KC31 and KC36, have highly anomalous phosphorous, common to rocks formed at the base of the continental slope (Sue Karl, personal commun., 1997). See table 1 for geochemical analyses and tables 2 through 6 for statistical data. 


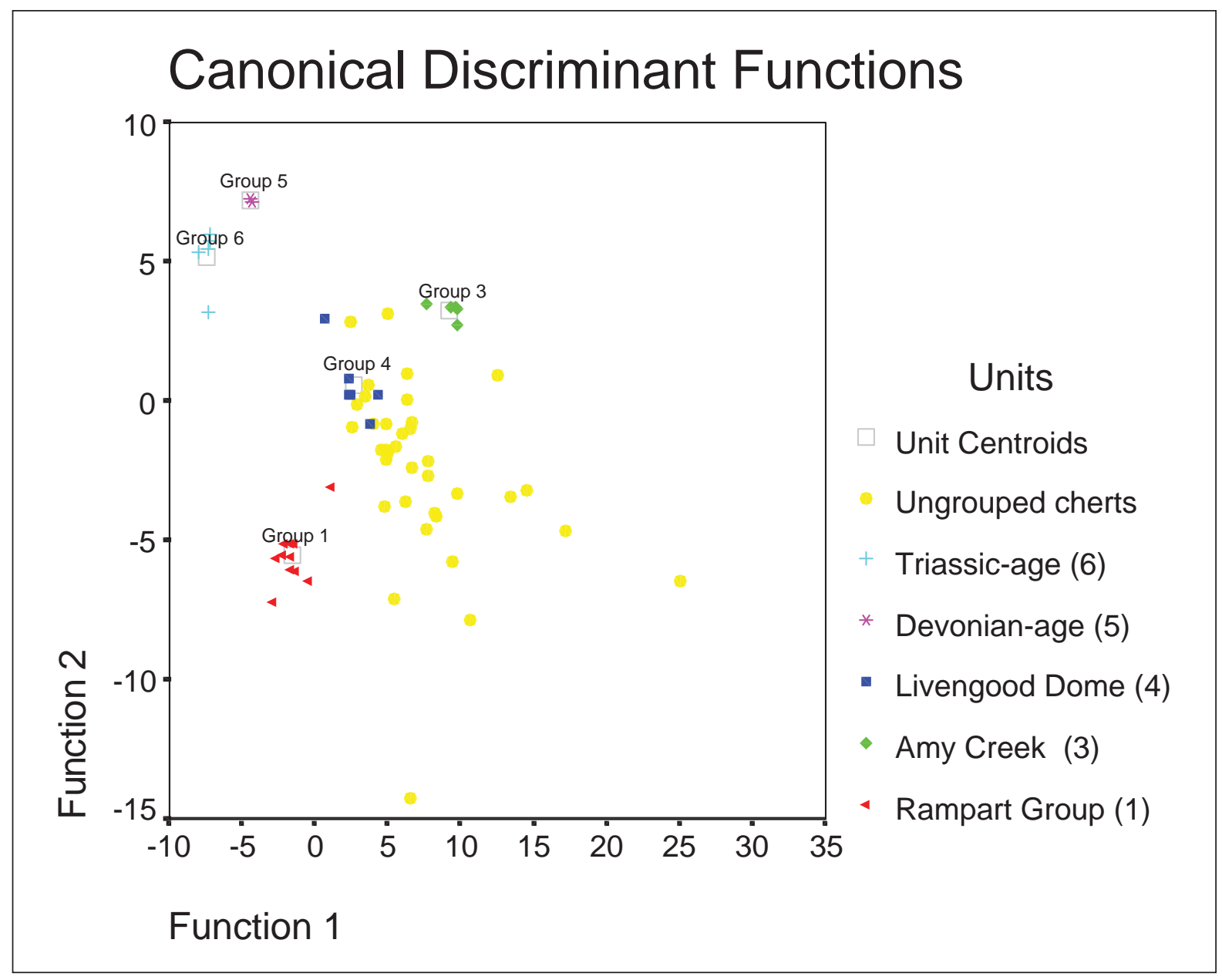

Figure 4. Unknown chert samples (excluding samples KC31 and KC36; see text). Function 1 and Function 2 are generated by the discriminant analysis and are listed in table 4 for each sample. The two outliers, KC31 and KC36, have highly anomalous phosphorous, common to rocks formed at the base of the continental slope (Sue Karl, personal commun., 1997). See table 1 for geochemical analyses and tables 2 through 6 for statistical data. 
Table 4. The hypothesized rock unit for each chert sample shown next to the predicted unit generated by the discriminant analysis. The hypothesized unit is based on sample characteristics and lithologic associations.

\begin{tabular}{|c|c|c|c|c|c|c|}
\hline Sample & $\begin{array}{l}\text { Hypothesized } \\
\text { Unit }\end{array}$ & Predicted & 1st Prob. & 2nd Prob. & Function1* & Function*2 \\
\hline JD169 & $\mathrm{L} ?$ & $\mathrm{~L}$ & $\mathrm{~L} / 1$ & $\mathrm{~A} / .0000$ & 4.9521 & -0.7805 \\
\hline JD46 & $\mathrm{L}$ ? & $\mathrm{L}$ & $\mathrm{L} / 1$ & $\mathrm{R} / .0000$ & 2.9101 & -0.0916 \\
\hline КС33 & $\mathrm{L} ?$ & $\mathrm{~L}$ & $\mathrm{~L} / 1$ & A/.0000 & 5.5187 & -1.5855 \\
\hline KC48 & $\mathrm{L} ?$ & $\mathrm{~L}$ & L/0.9227 & A/.0773 & 6.6415 & -0.7175 \\
\hline KC60 & L? & A & A/.5036 & $\mathrm{L} / .4964$ & 6.3422 & 1.0126 \\
\hline KC62 & $\mathrm{L} ?$ & A & $\mathrm{A} / .7907$ & L/.2903 & 8.3293 & -4.1132 \\
\hline KC67 & L? & A & $\mathrm{A} / .8943$ & L/.1057 & 8.2794 & -4.0013 \\
\hline RB37 & L? & $\mathrm{L}$ & L/1 & $\mathrm{A} / .0000$ & 5.065 & -1.8275 \\
\hline RB61 & $\mathrm{L}$ ? & $\mathrm{L}$ & $\mathrm{L} / 1$ & A/.0000 & 3.7293 & 0.6237 \\
\hline SL126 & $\mathrm{L}$ ? & $\mathrm{L}$ & $\mathrm{L} / 1$ & $\mathrm{~A} / .0000$ & 4.6168 & -1.7303 \\
\hline SL149 & $\mathrm{L} ?$ & $\mathrm{~L}$ & L/.9925 & A/.0075 & 6.6097 & -0.9541 \\
\hline SL151 & $\mathrm{L}$ ? & $\mathrm{L}$ & L/1 & $\mathrm{R} / .0000$ & 2.5396 & -0.8896 \\
\hline SL190 & $\mathrm{L} ?$ & A & $\mathrm{A} / 1$ & $\mathrm{~L} / .0000$ & 9.7917 & -3.3067 \\
\hline SL44 & $\mathrm{L} ?$ & $\mathrm{~L}$ & $\mathrm{~L} / 1$ & A/.0000 & 4.8719 & -1.7257 \\
\hline SL80 & $\mathrm{L}$ ? & $\mathrm{L}$ & L/.9942 & A/.0058 & 6.0588 & -1.1092 \\
\hline SL88 & $\mathrm{L} ?$ & A & A/.6395 & L/.3605 & 7.7571 & -2.1321 \\
\hline KC236 & $\mathrm{L} ?$ & $\mathrm{~L}$ & L/1 & L/1 & 2.5233 & 2.8937 \\
\hline SL79A & $\mathrm{A}$ ? & A & $\mathrm{A} / 1$ & L/.0000 & 9.4273 & -5.7065 \\
\hline JD175B & $\mathrm{A}$ ? & A & $\mathrm{A} / 1$ & $\mathrm{~A} / 1$ & 12.5831 & 0.9572 \\
\hline KC176 & A? & A & A/.9887 & L/.0113 & 6.3598 & 0.1134 \\
\hline KC47 & A? & A & $\mathrm{A} / 1$ & L/.0000 & 7.7975 & -2.658 \\
\hline КС98 & $A ?$ & A & $\mathrm{A} / 1$ & $\mathrm{~A} / 1$ & 25.0369 & -6.4501 \\
\hline RB111 & $\mathrm{A}$ ? & $\mathrm{L}$ & L/.9925 & A/.0075 & 5.0246 & 3.1642 \\
\hline RB121 & $\mathrm{A}$ ? & A & $\mathrm{A} / 1$ & $\mathrm{~A} / 1$ & 17.1892 & -4.6323 \\
\hline RB60 & $\mathrm{A}$ ? & A & A/.7618 & L/.2382 & 6.6416 & -2.3722 \\
\hline SL169 & A? & A & $\mathrm{A} / 1$ & $\mathrm{R} / .0000$ & 10.6421 & -7.8404 \\
\hline SL74C & $A ?$ & $\mathrm{~L}$ & $\mathrm{~L} / 1$ & $\mathrm{~A} / .0000$ & 3.4261 & 0.1758 \\
\hline SL78 & $A ?$ & A & $\mathrm{A} / 1$ & $\mathrm{~A} / 1$ & 14.6018 & -3.1858 \\
\hline SL74D & L?A? & $\mathrm{L}$ & $\mathrm{L} / 1$ & $\mathrm{~A} / .0000$ & 4.026 & -0.7625 \\
\hline RB115 & $\mathrm{L} ?$ & $\mathrm{~L}$ & L/1 & A/.0000 & 4.9499 & -2.0604 \\
\hline SL174 & $\mathrm{L}$ ? & $\mathrm{L}$ & L/.9972 & $\mathrm{R} / .0028$ & 4.7693 & -3.7573 \\
\hline RB130 & $?$ & $\mathrm{~L}$ & L/.9984 & A/.0016 & 6.2407 & -3.558 \\
\hline RR74B & $?$ & $\mathrm{R}$ & $\mathrm{R} / 1$ & $\mathrm{R} / 1$ & 6.5921 & -14.2 \\
\hline JD119 & $?$ & A & $\mathrm{A} / 1$ & $\mathrm{~A} / 1$ & 13.4427 & -3.39225 \\
\hline JD18 & $?$ & $\mathrm{R}$ & $\mathrm{R} / 1$ & $\mathrm{~L} / .0000$ & 5.4927 & -7.0479 \\
\hline КС63C & $?$ & $\mathrm{~L}$ & L/.9983 & A/.0017 & 7.642 & -4.537 \\
\hline КС31 & $A ?$ & $\mathrm{~T}$ & $\mathrm{~T} / 1$ & $\mathrm{~T} / 1$ & -64.9091 & 32.1456 \\
\hline КС36 & $A ?$ & $\mathrm{~T}$ & $\mathrm{~T} / 1$ & $\mathrm{~T} / 1$ & -144.3837 & 59.0735 \\
\hline
\end{tabular}

*These functions are plotted in figures 3 and 4.

$\mathrm{R}=$ Rampart unit $\quad \mathrm{R}$ ?=Hypothesized Rampart Group unit

A=Amy Creek unit ? ?=Hypothesized Amy Creek unit

L=Livengood Dome unit $\quad$ L?=Hypothesized Livengood Dome unit

$\mathrm{T}=$ Triassic-age unit 
units (Sue Karl, personal commun., 1997).

None of the unknown chert samples are assigned to the Devonian-age Troublesome Creek chert unit from the central Livengood Quadrangle. This may be due to the small sample size of the Devonian-age type locale samples, resulting in a very small range of geochemical signatures. Known Devonian cherts were not easily accessible and consequently were not abundantly sampled.

\section{K-MEANS CLUSTER ANALYSIS: METHODS AND RESULTS}

Using SPSS for Windows, K-Means cluster analysis was applied to the geochemical data to test whether this statistical method could find natural clusters in the data. Hierarchical cluster analysis using normalized geochemical data was also performed, but was found not to be any different from K-means cluster analysis using raw data. After trying K-means cluster analysis with many different numbers of clusters, eight clusters was found to be the most descriptive in clustering the samples, allowing for clusters to be distinguished.

\section{TYPE LOCALE CHERT SAMPLES}

K-means cluster analysis was first applied to the geochemical data of the type locale chert samples. Final cluster centers, an analysis of variance table, and the cluster classifications are found in table 5 . As shown in the cluster classification chart, the Rampart Group chert samples clearly do not cluster well into any one group. Amy Creek unit chert clusters well: three samples group together in cluster 3 , one sample groups with cluster 2 , and one sample groups with cluster 5 . The Livengood Dome chert unit clusters very well with all six samples grouping together in cluster 1 . The Devonian-age Troublesome Creek chert samples do not cluster together, but due to the small sample size this is not very significant. The Triassic-age chert samples cluster fairly well with two chert samples in cluster 1 and three samples in cluster 2. A large number of the samples from various rock units group into cluster 1 , indicating that the data as a whole, due to a lack of compositional uniformity, does not cluster as well into five distinct groups as discriminant analysis suggests.

\section{UNKNOWN CHERT SAMPLES}

K-means cluster analysis applied to the geochemical data of the samples of uncertain rock unit affinity was conducted in two ways. First, K-means cluster analysis was applied to the original data. Of the chert samples that were hypothesized to be Livengood Dome chert, all samples group together in cluster 1 . The hypothesized Amy Creek chert samples cluster in three different groups; two samples with cluster 2, two samples with cluster 4 , and seven samples with cluster 1 . Of the samples that are of unknown affinity two samples group with cluster 2 and eight samples group with cluster 1 (figs. 3 and 4).

The second method used the assignments given to the unknown chert samples by discriminant analysis previously conducted to see how well those assignments clustered. The cherts assigned to Rampart Group do not cluster well; one sample groups with cluster 8 , two samples group with cluster 2, two samples group with cluster 3 , two samples group with cluster 5, one sample groups with cluster 6 , and five samples group with cluster 1. Chert samples assigned to Amy Creek unit also do not cluster well, although slightly better than the Rampart Group: three samples group with cluster 2, two samples group with cluster 4, three samples group with cluster 3 , one sample groups with cluster 7 , and ten samples group with cluster 1. Chert samples assigned to Livengood Dome chert unit cluster well; two samples group with cluster 2 , and 24 samples group with cluster 1 . The Devonian-age Troublesome Creek chert samples only have two assignments, making the sample size too small to make generalizations. The chert samples assigned to the Triassic-age unit cluster well; three samples group with cluster 2, and four samples group with cluster 1.

Table 6 displays the K-means cluster analysis of original data from chert samples of unknown units, the final cluster centers, analysis of variance (ANOVA) data, and cluster classifications of samples. Table 7 shows K-means cluster analysis of discriminant analysis data, final cluster centers, and ANOVA. 
Table 5. K-means cluster analysis of type locale chert data, final Cluster Centers

\begin{tabular}{|c|c|c|c|c|c|c|c|c|}
\hline & Cluster 1 & Cluster 2 & Cluster 3 & Cluster 4 & Cluster 5 & Cluster 6 & Cluster 7 & Cluster 8 \\
\hline $\mathrm{Al}_{2} \mathrm{O}_{3}$ & 2.5 & 3.9 & 3.7 & 5.2 & 2.3 & 6.5 & 8.1 & 10.9 \\
\hline $\mathrm{Ba}$ & 108.2 & 369.3 & 1223.2 & 2122.7 & 4938.0 & 7165.5 & 2881.4 & 2606.8 \\
\hline $\mathrm{CaO}$ & .46 & .03 & .19 & .21 & .00 & .66 & .72 & .06 \\
\hline $\mathrm{Ce}$ & 14.9 & 15.4 & 4.6 & 18.4 & 14.9 & 9.0 & 2.5 & 17.3 \\
\hline $\mathrm{Cr}$ & 7.8 & 9.9 & 3.9 & 13.8 & .00 & 173.3 & 15.2 & 8.3 \\
\hline $\mathrm{Fe}_{2} \mathrm{O}_{3}$ & 1.7 & 2.9 & 1.5 & 2.9 & .24 & 2.5 & 4.4 & 4.4 \\
\hline $\mathrm{K}_{2} \mathrm{O}$ & .25 & .51 & .40 & 1.1 & .22 & 1.1 & 1.3 & 2.3 \\
\hline $\mathrm{La}$ & 4.0 & 7.0 & 2.3 & 7.2 & 15.2 & 15.6 & 7.9 & 5.4 \\
\hline $\mathrm{MgO}$ & .75 & 1.4 & 1.3 & 2.5 & .19 & 3.7 & 2.4 & 3.8 \\
\hline $\mathrm{MnO}$ & .08 & .12 & .03 & .07 & .00 & .09 & .40 & .12 \\
\hline $\mathrm{Na}_{2} \mathrm{O}$ & .58 & .39 & .59 & .07 & .00 & .93 & .21 & .72 \\
\hline $\mathrm{P}_{2} \mathrm{O}_{5}$ & .03 & .06 & .04 & .04 & .76 & .54 & .06 & .05 \\
\hline $\mathrm{Rb}$ & 9.3 & 15.2 & 13.4 & 44.0 & 9.8 & 18.5 & 41.6 & 70.4 \\
\hline $\mathrm{SiO} \mathrm{F}_{2}$ & 93.2 & 90.0 & 91.6 & 87.4 & 96.4 & 81.7 & 81.0 & 76.4 \\
\hline $\mathrm{Sr}$ & 28.6 & 15.4 & 29.6 & 12.7 & 134.5 & 69.5 & 22.4 & 21.9 \\
\hline $\mathrm{TiO}{ }_{2}$ & .1371 & .18 & .14 & .22 & .11 & .30 & .37 & .45 \\
\hline $\mathrm{V}$ & 25.8 & 71.3 & 76.9 & 82.0 & 157.5 & 180.3 & 60.3 & 115.8 \\
\hline $\mathrm{Y}$ & 6.0 & 8.5 & 7.1 & 10.1 & 47.7 & 48.5 & 19.7 & 10.4 \\
\hline $\mathrm{Zr}$ & 36.8 & 51.6 & 38.6 & 54.8 & 24.7 & 83.6 & 70.5 & 111.9 \\
\hline
\end{tabular}

Highlighted cells are element values that characterize the particular cluster.

Cluster Classification:

\begin{tabular}{|c|c|c|c|c|c|c|c|c|c|}
\hline & $\begin{array}{c}\text { Cluster } \\
1\end{array}$ & $\begin{array}{c}\text { Cluster } \\
2\end{array}$ & $\begin{array}{c}\text { Cluster } \\
3\end{array}$ & $\begin{array}{c}\text { Cluster } \\
4\end{array}$ & $\begin{array}{c}\text { Cluster } \\
5\end{array}$ & $\begin{array}{c}\text { Cluster } \\
6\end{array}$ & $\begin{array}{c}\text { Cluster } \\
7\end{array}$ & $\begin{array}{c}\text { Cluster } \\
8\end{array}$ & Total \\
\hline Rampart Group & 3 samples & 2 & 2 & 1 & & 1 & 1 & 1 & 11 \\
\hline Amy Creek & & 1 & 3 & & 1 & & & & 5 \\
\hline Livengood Dome & 6 & & & & & & & & 6 \\
\hline Devonian-age & 1 & & & 1 & & & & & 2 \\
\hline Triassic-age & 2 & 3 & & & & & & & 5 \\
\hline Total & 12 & 6 & 5 & 2 & 1 & 1 & 1 & 1 & 29 \\
\hline
\end{tabular}

Analysis of Variance (ANOVA)
\begin{tabular}{|c|c|c|c|c|c|c|}
\hline Variable & Cluster MS & DF & Error MS & DF & F & Prob. \\
\hline $\mathrm{Al}_{2} \mathrm{O}_{3}$ & 14.6700 & 7 & 10.342 & 21 & 1.4184 & .250 \\
\hline $\mathrm{Ba}$ & 10590625.3902 & 7 & 5020.674 & 21 & 2109.4030 & .000 \\
\hline $\mathrm{CaO}$ & .1788 & 7 & .469 & 21 & .3805 & .303 \\
\hline $\mathrm{Ce}$ & 91.4051 & 7 & 252.545 & 21 & .3619 & .914 \\
\hline $\mathrm{Cr}$ & 3811.8904 & 7 & 131.311 & 21 & 29.0294 & .000 \\
\hline $\mathrm{Fe}_{2} \mathrm{O}_{3}$ & 3.2977 & 7 & 3.975 & 21 & .8296 & .575 \\
\hline $\mathrm{K}_{2} \mathrm{O}$ & .8044 & 7 & .181 & 21 & 4.4429 & .004 \\
\hline $\mathrm{La}$ & 42.4401 & 7 & 36.625 & 21 & 1.1588 & .367 \\
\hline $\mathrm{MgO}$ & 2.9663 & 7 & 1.103 & 21 & 2.6870 & .037 \\
\hline $\mathrm{MnO}_{\mathrm{Na}} \mathrm{O}$ & .0187 & 7 & .010 & 21 & 1.8032 & .140 \\
\hline $\mathrm{P}_{2} \mathrm{O}_{5}$ & .1605 & 7 & .730 & 21 & .2196 & .977 \\
\hline $\mathrm{Rb}$ & .1009 & 7 & .002 & 21 & 38.4377 & .000 \\
\hline $\mathrm{SiO} \mathrm{S}_{2}$ & 73.9582 & 7 & 246.988 & 21 & 3.3360 & .015 \\
\hline $\mathrm{Sr}$ & 2069.6027 & 7 & 590.047 & 21 & 3.5075 & .015 \\
\hline $\mathrm{TiO} \mathrm{N}_{2}$ & .0226 & 7 & .028 & 21 & .8018 & .012 \\
\hline $\mathrm{V}$ & 6303.1727 & 7 & 30.716 & 21 & 15.0360 & .595 \\
\hline $\mathrm{Y}$ & 461.8463 & 7 & 30.716 & 21 & 15.0360 & .000 \\
\hline $\mathrm{Zr}$ & 1203.5393 & 7 & 2649.203 & 21 & .4543 & .000 \\
\hline
\end{tabular}

Highlighted elements show significant variability between clusters at the 95 percent confidence level 
Table 6. K-means cluster analysis of original data, chert samples from unknown units, final Cluster Centers.

\begin{tabular}{|c|c|c|c|c|c|c|c|c|}
\hline & Cluster 1 & Cluster 2 & Cluster 3 & Cluster 4 & Cluster 5 & Cluster 6 & Cluster 7 & Cluster 8 \\
\hline $\mathrm{Al}_{2} \mathrm{O}_{3}$ & 1.9 & 3.2 & 3.7 & 3.4 & 9.5 & 5.2 & 2.3 & 6.5 \\
\hline $\mathrm{Ba}$ & 92.5 & 399.6 & 1223.2 & 919.4 & 2744.1 & 2122.7 & 4938.0 & 7165.5 \\
\hline $\mathrm{CaO}$ & .41 & .07 & .19 & .04 & .39 & .21 & .00 & .66 \\
\hline $\mathrm{Ce}$ & 14.5 & 16.0 & 4.6 & 4.7 & 9.9 & 18.4 & 14.9 & 9.0 \\
\hline $\mathrm{Cr}$ & 4.8 & 7.4 & 3.9 & .00 & 11.8 & 13.8 & .00 & 173.3 \\
\hline $\mathrm{Fe}_{2} \mathrm{O}_{3}$ & .80 & 2.0 & 1.5 & .69 & 4.4 & 2.9 & .24 & 2.5 \\
\hline $\mathrm{K}_{2} \mathrm{O}$ & .30 & .48 & .40 & .69 & 1.8 & 1.1 & .22 & 1.1 \\
\hline $\mathrm{La}$ & 3.3 & 5.4 & 2.3 & 3.4 & 6.6 & 7.2 & 15.2 & 15.6 \\
\hline $\mathrm{MgO}$ & .47 & 1.0 & 1.3 & .62 & 3.1 & 2.5 & .19 & 3.7 \\
\hline $\mathrm{MnO}$ & .04 & .08 & .03 & .00 & .26 & .07 & .00 & .09 \\
\hline $\mathrm{Na}_{2} \mathrm{O}$ & .18 & .24 & .59 & .01 & .46 & .07 & .00 & .93 \\
\hline $\mathrm{P}_{2} \mathrm{O}_{5}$ & .24 & .09 & .04 & .14 & .06 & .04 & .76 & .54 \\
\hline $\mathrm{Rb}$ & 8.7 & 13.2 & 13.4 & 16.4 & 56.0 & 44.0 & 9.8 & 18.5 \\
\hline $\mathrm{SiO}{ }_{2}$ & 95.1 & 92.5 & 91.6 & 95.0 & 78.7 & 87.4 & 96.4 & 81.7 \\
\hline $\mathrm{Sr}$ & 16.6 & 15.0 & 29.6 & 13.8 & 22.1 & 12.7 & 134.5 & 69.5 \\
\hline $\mathrm{TiO} \mathrm{F}_{2}$ & .08 & .14 & .14 & .11 & .41 & .22 & .11 & .30 \\
\hline $\mathrm{V}$ & 28.0 & 61.9 & 76.9 & 150.1 & 88.0 & 82.0 & 157.5 & 180.3 \\
\hline $\mathrm{Y}$ & 6.5 & 7.9 & 7.1 & 5.9 & 15.0 & 10.1 & 47.7 & 48.5 \\
\hline $\mathrm{Zr}$ & 16.9 & 36.1 & 38.6 & 18.3 & 91.2 & 54.8 & 24.7 & 83.6 \\
\hline
\end{tabular}

Highlighted cells are element values which characterize the particular cluster.

Cluster Classification:

\begin{tabular}{|c|c|c|c|c|c|c|c|c|c|}
\hline & $\begin{array}{c}\text { Cluster } \\
1\end{array}$ & $\begin{array}{c}\text { Cluster } \\
2\end{array}$ & $\begin{array}{c}\text { Cluster } \\
3\end{array}$ & $\begin{array}{c}\text { Cluster } \\
4\end{array}$ & $\begin{array}{c}\text { Cluster } \\
5\end{array}$ & $\begin{array}{c}\text { Cluster } \\
6\end{array}$ & $\begin{array}{c}\text { Cluster } \\
7\end{array}$ & $\begin{array}{c}\text { Cluster } \\
8\end{array}$ & Total \\
\hline Rampart Group & 3 & 2 & 2 & & 2 & 1 & & 1 & 11 \\
\hline Amy Creek & & 1 & 3 & & & & 1 & & 5 \\
\hline Livengood & 6 & & & & & & & & 6 \\
\hline Devonian-age & 1 & & & & & 1 & & & 2 \\
\hline Triassic-age & 2 & 3 & & & & & & & 5 \\
\hline Hypothesized Liv & 17 & & & & & & & & 17 \\
\hline Hypothesized Amy & 7 & 2 & & 2 & & & & & 11 \\
\hline Unknown unit chert & 8 & 2 & & & & & & & 10 \\
\hline Total & 44 & 10 & 5 & 2 & 2 & 2 & 1 & 1 & 67 \\
\hline
\end{tabular}

Analysis of Variance (ANOVA)

\begin{tabular}{|c|c|c|c|c|c|c|}
\hline Variable & Cluster MS & DF & Error MS & DF & F & Prob. \\
\hline $\mathrm{Al}_{2} \mathrm{O}_{3}$ & 22.5801 & 7 & 5.108 & 59.0 & 4.4203 & .001 \\
\hline $\mathrm{Ba}$ & 12793546.7485 & 7 & 5843.948 & 59.0 & 2189.1956 & .000 \\
\hline $\mathrm{CaO}$ & .2048 & 7 & 1.421 & 59.0 & .1440 & .994 \\
\hline $\mathrm{Ce}$ & 108.0647 & 7 & 220.859 & 59.0 & .4893 & .839 \\
\hline $\mathrm{Cr}$ & 4022.6693 & 7 & 93.883 & 59.0 & 42.8476 & .000 \\
\hline $\mathrm{Fe}_{2} \mathrm{O}_{3}$ & 6.0746 & 7 & 1.954 & 59.0 & 3.1083 & .007 \\
\hline $\mathrm{K}_{2} \mathrm{O}$ & .8429 & 7 & .123 & 59.0 & 6.8070 & .000 \\
\hline $\mathrm{La}$ & 50.2703 & 7 & 45.115 & 59.0 & 1.1143 & .366 \\
\hline $\mathrm{MgO}$ & 4.5446 & 7 & .564 & 59.0 & 8.0457 & .000 \\
\hline $\mathrm{MnO}$ & .0161 & 7 & .006 & 59.0 & 2.4600 & .028 \\
\hline $\mathrm{Na}_{2} \mathrm{O}$ & .2241 & 7 & .314 & 59.0 & .7132 & .661 \\
\hline $\mathrm{P}_{2} \mathrm{O}_{5}$ & .1191 & 7 & .799 & 59.0 & .1491 & .993 \\
\hline $\mathrm{Rb}$ & 928.2761 & 7 & 129.649 & 59.0 & 7.1599 & .000 \\
\hline $\mathrm{SiO}_{2}$ & 116.3523 & 7 & 26.159 & 59.0 & 4.4478 & .001 \\
\hline $\mathrm{Sr}$ & 2422.8500 & 7 & 616.478 & 59.0 & 3.9301 & .001 \\
\hline $\mathrm{TiO} \mathrm{O}_{2}$ & .0431 & 7 & .012 & 59.0 & 3.3955 & .004 \\
\hline $\mathrm{V}$ & 11375.1142 & 7 & 1301.690 & 59.0 & 8.7387 & .000 \\
\hline $\mathrm{Y}$ & 489.1197 & 7 & 168.833 & 59.0 & 2.8971 & .011 \\
\hline $\mathrm{Zr}$ & 2720.3168 & 7 & 1169.045 & 59.0 & 2.3270 & .036 \\
\hline
\end{tabular}

Highlighted elements show significant variability between clusters at the 95 percent confidence level. 
Table 7. K-means cluster analysis of discriminant analysis data, final Cluster Centers

\begin{tabular}{|c|c|c|c|c|c|c|c|c|}
\hline & Cluster 1 & Cluster 2 & Cluster 3 & Cluster 4 & Cluster 5 & Cluster 6 & Cluster 7 & Cluster 8 \\
\hline $\mathrm{Al}_{2} \mathrm{O}_{3}$ & 1.9 & 3.2 & 3.7 & 3.4 & 9.5 & 5.2 & 2.3 & 6.5 \\
\hline $\mathrm{Ba}$ & 92.5 & 399.6 & 1223.2 & 919.4 & 2744.1 & 2122.7 & 4938.0 & 7165.5 \\
\hline $\mathrm{CaO}$ & .41 & .07 & .19 & .04 & .39 & .21 & .00 & .66 \\
\hline $\mathrm{Ce}$ & 14.5 & 16.0 & 4.6 & 4.7 & 9.9 & 18.4 & 14.9 & 9.0 \\
\hline $\mathrm{Cr}$ & 4.8 & 7.4 & 3.9 & .00 & 11.8 & 13.8 & .00 & 173.3 \\
\hline $\mathrm{Fe}_{2} \mathrm{O}_{3}$ & .80 & 2.0 & 1.5 & .69 & 4.4 & 2.9 & .24 & 2.5 \\
\hline $\mathrm{K}_{2} \mathrm{O}$ & .30 & .48 & .40 & .69 & 1.8 & 1.1 & .22 & 1.1 \\
\hline $\mathrm{La}$ & 3.3 & 5.4 & 2.3 & 3.4 & 6.6 & 7.2 & 15.2 & 15.6 \\
\hline $\mathrm{MgO}$ & .47 & 1.0 & 1.3 & .62 & 3.1 & 2.5 & .19 & 3.7 \\
\hline $\mathrm{MnO}$ & .04 & .08 & .03 & .00 & .26 & .07 & .00 & .09 \\
\hline $\mathrm{Na}_{2} \mathrm{O}$ & .18 & .24 & .59 & .01 & .46 & .07 & .00 & .93 \\
\hline $\mathrm{P}_{2} \mathrm{O}_{5}$ & .24 & .09 & .04 & .14 & .06 & .04 & .76 & .54 \\
\hline $\mathrm{Rb}$ & 8.7 & 13.2 & 13.4 & 16.4 & 56.0 & 44.0 & 9.8 & 18.5 \\
\hline $\mathrm{SiO}_{2}$ & 95.1 & 92.5 & 91.6 & 95.0 & 78.7 & 87.4 & 96.4 & 81.7 \\
\hline $\mathrm{Sr}$ & 16.6 & 15.0 & 29.6 & 13.8 & 22.1 & 12.7 & 134.5 & 69.5 \\
\hline $\mathrm{TiO} \mathrm{O}_{2}$ & .08 & .14 & .14 & .11 & .41 & .22 & .11 & .30 \\
\hline $\mathrm{V}$ & 28.0 & 61.9 & 76.9 & 150.1 & 88.0 & 82.0 & 157.5 & 180.3 \\
\hline $\mathrm{Y}$ & 6.5 & 7.9 & 7.1 & 5.9 & 15.0 & 10.1 & 47.7 & 48.5 \\
\hline $\mathrm{Zr}$ & 16.9 & 36.1 & 38.6 & 18.3 & 91.2 & 54.8 & 24.7 & 84.0 \\
\hline
\end{tabular}

Highlighted cells are element values which characterize the particular cluster.

Cluster Classification:

\begin{tabular}{|l|c|c|c|c|c|c|c|c|c|} 
& Cluster & Cluster & Cluster & Cluster & Cluster & Cluster & Cluster & Cluster & Total \\
& $\mathbf{1}$ & $\mathbf{2}$ & $\mathbf{3}$ & $\mathbf{4}$ & $\mathbf{5}$ & $\mathbf{6}$ & $\mathbf{7}$ & $\mathbf{8}$ & $\mathbf{1}$ \\
\hline Rampart Group & 5 & 2 & 2 & & 2 & 1 & & 1 & $\mathbf{1 3}$ \\
\hline Amy Creek & 10 & 3 & 3 & 2 & & & 1 & & $\mathbf{1 9}$ \\
\hline Livengood & 24 & 2 & & & & & & & $\mathbf{2 6}$ \\
\hline Devonian-age & 1 & & & & & 1 & & & $\mathbf{2}$ \\
\hline Triassic-age & 4 & 3 & & & & & & & $\mathbf{7}$ \\
\hline \multicolumn{1}{r|}{ Total } & $\mathbf{4 4}$ & $\mathbf{1 0}$ & $\mathbf{5}$ & $\mathbf{2}$ & $\mathbf{2}$ & $\mathbf{2}$ & $\mathbf{1}$ & $\mathbf{1}$ & $\mathbf{6 7}$ \\
\hline
\end{tabular}

Analysis of Variance (ANOVA)
\begin{tabular}{|c|c|c|c|c|c|c|}
\hline Variable & Cluster MS & DF & Error MS & DF & F & Prob. \\
\hline $\mathrm{Al}_{2} \mathrm{O}_{3}$ & 22.5801 & 7 & 5.108 & 59.0 & 4.4203 & .001 \\
\hline $\mathrm{Ba}$ & 12793546.7485 & 7 & 5843.948 & 59.0 & 2189.1956 & .000 \\
\hline $\mathrm{CaO}$ & .2048 & 7 & 1.421 & 59.0 & .1440 & .994 \\
\hline $\mathrm{Ce}$ & 108.0647 & 7 & 220.859 & 59.0 & .4893 & .839 \\
\hline $\mathrm{Cr}$ & 4022.6693 & 7 & 93.883 & 59.0 & 42.8476 & .000 \\
\hline $\mathrm{Fe}_{2} \mathrm{O}_{3}$ & 6.0746 & 7 & 1.954 & 59.0 & 3.1083 & .007 \\
\hline $\mathrm{K}_{2} \mathrm{O}$ & .8429 & 7 & .123 & 59.0 & 6.8070 & .000 \\
\hline $\mathrm{La}$ & 50.2703 & 7 & 45.115 & 59.0 & 1.1143 & .366 \\
\hline $\mathrm{MgO}$ & 4.5446 & 7 & .564 & 59.0 & 8.0457 & .000 \\
\hline $\mathrm{MnO}$ & .0161 & 7 & .006 & 59.0 & 2.4600 & .028 \\
\hline $\mathrm{Na}_{2} \mathrm{O}$ & .2241 & 7 & .314 & 59.0 & .7132 & .661 \\
\hline $\mathrm{P}_{2} \mathrm{O}_{5}$ & .1191 & 7 & .799 & 59.0 & .1491 & .993 \\
\hline $\mathrm{Rb}$ & 928.2761 & 7 & 129.649 & 59.0 & 7.1599 & .000 \\
\hline $\mathrm{SiO}{ }_{2}$ & 116.3523 & 7 & 26.159 & 59.0 & 4.4478 & .001 \\
\hline $\mathrm{Sr}$ & 2422.8500 & 7 & 616.478 & 59.0 & 3.9301 & .001 \\
\hline $\mathrm{TiO}{ }_{2}$ & .0431 & 7 & .012 & 59.0 & 3.3955 & .004 \\
\hline $\mathrm{V}$ & 11375.1142 & 7 & 1301.690 & 59.0 & 8.7387 & .000 \\
\hline $\mathrm{Y}$ & 489.1197 & 7 & 168.833 & 59.0 & 2.8971 & .011 \\
\hline $\mathrm{Zr}$ & 2720.3168 & 7 & 1169.045 & 59.0 & 2.3270 & .036 \\
\hline
\end{tabular}

\footnotetext{
Highlighted elements are significant at the 95 percent confidence level.
} 


\section{CONCLUSIONS}

Discriminant analysis of the type locale cherts results in data that distinguish between the five different rock units, verifying that discriminant analysis is a viable means for differentiating between our chert units based on geochemistry. Applying discriminant analysis to both the major oxide and minor element data results in better correlation between the type locale cherts and the predicted units than major or minor element analysis alone. However, simple plots such as a plot of vanadium versus magnesium oxide (fig. 5) in most cases shows pronounced and distinct differences between chert samples from our different known rock units. These types of ratios can be calculated using modern principal component analysis (PCA) and then tested to evaluate potential statistical groupings.

K-means cluster analysis suggests that the Rampart Group chert unit is heterogeneous in nature and does not cluster well into any one group. All the Rampart samples were collected from the area previously mapped as Rampart Group in the Tanana B-1 Quadrangle (Reifenstuhl and others, 1997; Wilson and others, 1998) on the basis of the associated gabbros and basalts. Due to the structural complexities and numerous faults in the area, the heterogeneous nature of the Rampart Group cherts suggested by cluster analysis may indicate that some of the chert samples previously mapped as Rampart Group cherts are in fact not Rampart Group cherts, but fault slivers from other units.

The K-means cluster analysis is most applicable in differentiating the Livengood Dome chert unit, which clusters very well, suggesting a homogenous nature. Chert sample KC317, which was collected from the Yukon Territory, Canada, from similar Ordovician-age stratigraphy and with similar lithologic associations, falls into the Livengood chert unit, indicating a long-distance correlation. In contrast, discriminant analysis differentiates into the five distinct units, but the difference between Livengood Dome unit chert and Amy Creek unit chert is often difficult to assess based on chemical analysis alone.

The tendency for the Amy Creek chert unit and the Triassic-age chert unit to form distinct compositional clusters is between that of Livengood and Rampart. Consequently, discriminant analysis is a generally useful tool in assigning unknown chert samples to those units.

In general, when discriminant analysis is applied and the data are assigned to predetermined groupings, there is little difficulty assigning each sample to a group. In contrast, when K-means cluster analysis is applied, creating natural clusters, it is more difficult to distinguish between different groups.

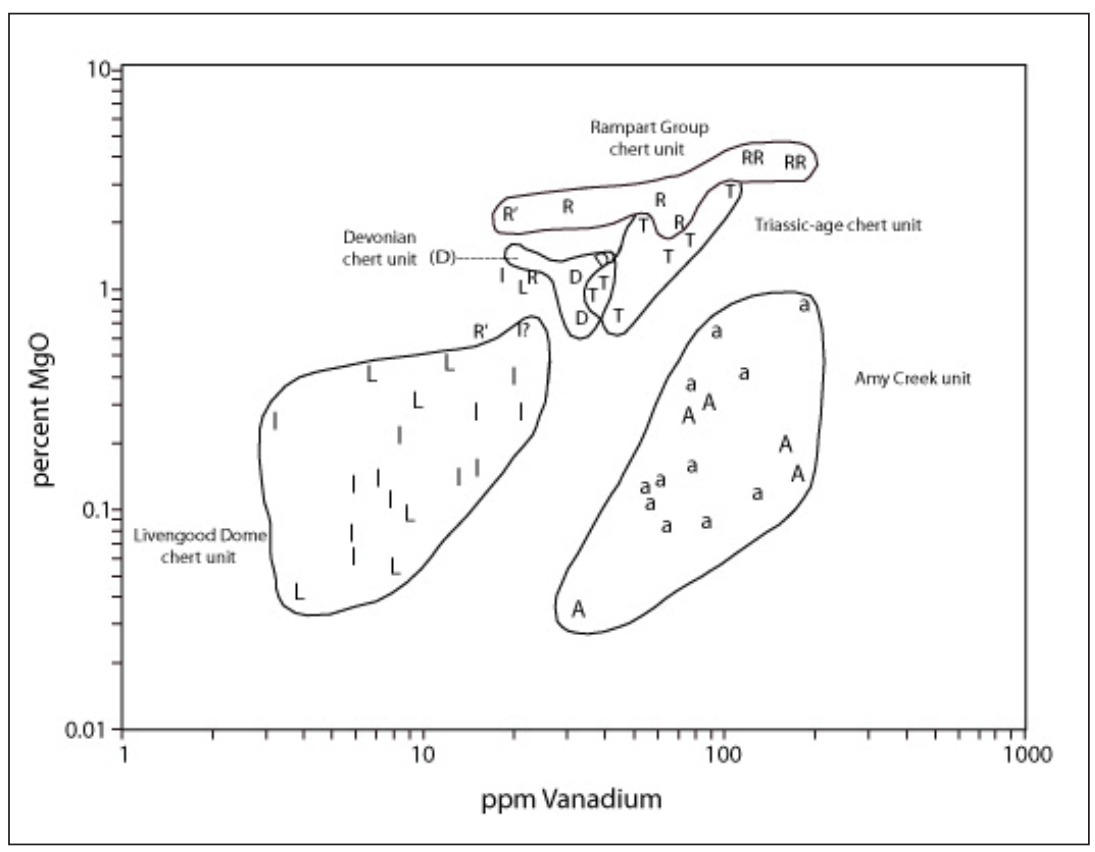

Figure 5. Plot of magnesium oxide ( $\mathrm{MgO}$ ) versus vanadium $(\mathrm{V})$ for chert samples. $R=$ Rampart Group type locale chert, $R^{\prime}=$ hypothesized Rampart Group chert, $T=$ Triassic-age chert, $D=$ Devonian-age Troublesome Creek chert, $L=$ Livengood Dome type locale chert, l=hypothesized Livengood Dome chert, A=Amy Creek type locale chert, a=hypothesized Amy Creek chert. See table 1 for geochemical data. 
Our pilot study suggests that chert samples from our five mappable units generally have distinguishable geochemical signatures. However, all units, and especially the Devonian unit, must be carefully considered due to small sample size, which may or may not be representative of the entire unit. Correlations generated by discriminant analysis and K-means cluster analysis are useful, but should be tempered with geologic field relationships, lithologic associations, and considerations of the original depositional environment. We encourage others to further test the application of this potentially powerful tool.

\section{ACKNOWLEDGMENTS}

We thank Jacob Mongrain (DGGS and UAF), and De Anne Stevens (DGGS) for reviewing this report, and Andrea Loveland for GIS and metadata assistance.

\section{REFERENCES}

Chapman, R.M., Yeend, Warren, Brosgé, W.P., and Reiser, H.N., 1982, Reconnaissance geologic map of the Tanana Quadrangle, Alaska: U.S. Geological Survey Open-File Report 82-734, 18 p., 1 sheet, scale 1:250,000.

Dover, J.H., 1994, Geology of part of east-central Alaska, in Plafker, G., and Berg, H.C., eds., The Geology of Alaska: Boulder, Colorado, Geological Society of America, The Geology of North America, v. G-1.

Emery, K.O., 1970, Continental margins of the world, Geology of the east Atlantic continental margin, General Economic papers ICSU/SCOF Working Party 31 Symposium: Cambridge, Report No. 70/13, p. 7-29.
Jones, D.L., and Murchey, Benita, 1986, Geologic significance of Paleozoic and Mesozoic radiolarian chert: Annual Review of Earth and Planetary Science, v. 14, p. 455-492.

Murray, Richard W., Buchholtz ten Brink, Marilyn R., Gerlach, David C., Russ, G. Price III, Jones, David L., 1992, Rare earth, major, and trace element composition of Monterey and DSDP chert and associated host sediment: Assessing the influence of chemical fractionation during diagenesis: Geochimica et Cosmochimica Acta, v. 56, no. 7, p.2,657-2,671.

Reifenstuhl, R.R., Dover, J.H., Pinney, D.S., Newberry, R.J., Clautice, K.H., Liss, S.A., Blodgett, R.B., Bundtzen, T.K., and Weber, F.R., 1997, Geologic map of the Tanana B-1 Quadrangle, central Alaska: Alaska Division of Geological \& Geophysical Surveys Report of Investigations 97-15a 17 p., 1 sheet, scale 1:63,360.

Weber, F.R., Wheeler, K.L., Rinchart, C.D., Chapman, R.M., and Blodgett, R.B., 1992, Geologic map of the Livengood quadrangle, Alaska: U.S. Geological Survey Open-file Report 92-562, 20 p., 1 sheet, scale 1:250,000.

Wilson, F.H., Dover, J.H., Bradley, D.C., Weber, F.R., Bundtzen, T.K., and Haeussler, P.J., 1998, Geologic map of central (Interior) Alaska, U.S. Geological Survey Open-file Report 98-133-A, Version 1.286 p., 1 sheet (scale 1:500,000). 